\title{
Superficial Layers Suppress the Deep Layers to Fine-tune Cortical Coding
}

\author{
(DScott R. Pluta, ${ }^{1,2}$ @Greg I. Telian, ${ }^{2}{ }^{\circledR}$ Alexander Naka, ${ }^{2}$ and ${ }^{\circledR}$ Hillel Adesnik $^{2}$ \\ ${ }^{1}$ Department of Molecular and Cell Biology and the Helen Wills Neuroscience Institute, University of California, Berkeley, California 94720, and \\ ${ }^{2}$ Department of Biology, Purdue University, West Lafayette, Indiana 47907
}

The descending microcircuit from layer $2 / 3$ (L2/3) to layer 5 (L5) is one of the strongest excitatory pathways in the cortex, presumably forming a core component of its feedforward hierarchy. To date, however, no experiments have selectively tested the impact of L2/3 activity on L5 during active sensation. We used optogenetic, cell-type-specific manipulation of L2/3 neurons in the barrel cortex of actively sensing mice (of either sex) to elucidate the significance of this pathway to sensory coding in L5. Contrary to standard models, activating L2/3 predominantly suppressed spontaneous activity in L5, whereas deactivating L2/3 mainly facilitated touch responses in L5. Somatostatin interneurons are likely important to this suppression because their optogenetic deactivation significantly altered the functional impact of $\mathrm{L} 2 / 3$ onto $\mathrm{L} 5$. The net effect of $\mathrm{L} 2 / 3$ was to enhance the stimulus selectivity and expand the range of $\mathrm{L} 5$ output. These data imply that the core cortical pathway increases the selectivity and expands the range of cortical output through feedforward inhibition.

Key words: active sensing; circuits; cortex; receptive fields; sensory coding; translaminar

\section{Significance Statement}

The primary sensory cortex contains six distinct layers that interact to form the basis of our perception. While rudimentary patterns of connectivity between the layers have been outlined quite extensively in vitro, functional relationships in vivo, particularly during active sensation, remain poorly understood. We used cell-type-specific optogenetics to test the functional relationship between layer $2 / 3$ and layer 5 . Surprisingly, we discovered that L2/3 primarily suppresses cortical output from L5. The recruitment of somatostatin-positive interneurons is likely fundamental to this relationship. The net effect of this translaminar suppression is to enhance the selectivity and expand the range of receptive fields, therefore potentially sharpening the perception of space.

\section{Introduction}

Identifying the circuits that contribute to the activity of L5 cortical projection neurons is essential for understanding cortical computation and its role in sensation and behavior. Extensive research has identified a descending connection from pyramidal cells (PCs) in L2/3 as a prominent source of intracortical excitation to L5 (Lefort et al., 2009; Otsuka and Kawaguchi, 2009; Petreanu et al., 2009; Adesnik and Scanziani, 2010; Hooks et al.,

Received June 8, 2018; revised Dec. 17, 2018; accepted Dec. 20, 2018.

Author contributions: S.R.P. and H.A. edited the paper; S.R.P. and H.A. designed research; S.R.P. and A.N. performed research; G.I.T. contributed unpublished reagents/analytic tools; S.R.P. and A.N. analyzed data; S.R.P. and H.A. wrote the paper.

This work was supported by New York Stem Cell Foundation and National Institute of Neurological Disorders and Stroke Grant DP2NS087725-01. A.N. was supported by National Institutes of Health National Research Service Award F31NS093925. G.I.T. was supported by the Howard Hughes Medical Institute Gilliam Fellowship. H.A. is a New York Stem Cell Robertson Investigator. We thank Dan Feldman, members of the H.A. laboratory, and Alex Chubykin for comments on the manuscript.

The authors declare no competing financial interests.

Correspondence should be addressed to Hillel Adesnik at hadesnik@berkeley.edu.

https://doi.org/10.1523/JNEUROSCI.1459-18.2018

Copyright $\odot 2019$ the authors $\quad 0270-6474 / 19 / 392052-13 \$ 15.00 / 0$
2011; Feldmeyer, 2012). A common assumption from these data is that $\mathrm{L} 2 / 3$ provides a critical component of sensory facilitation to $\mathrm{L} 5$, and response latencies in these two layers to whisker deflection are partly consistent with this notion (Armstrong-James et al., 1992). Nonetheless, pharmacological or cryogenic deactivation experiments in vivo argue that $\mathrm{L} 2 / 3$ has no effect on sensory responses in L5 (Schwark et al., 1986; Constantinople and Bruno, 2013). However, these prior techniques had limited temporal precision and were performed in anesthetized or sedated animals, where spontaneous and interneuron activity is substantially altered (de Kock and Sakmann, 2008; Constantinople and Bruno, 2011; Adesnik et al., 2012; Sellers et al., 2013; McGinley et al., 2015). To overcome these technical limitations, L2/3-specific optogenetic manipulations in awake, behaving animals are needed. Previously, L2/3 photoactivation in brain slices suggested that L2/3 could modulate spiking in L5 neurons either up or down (Adesnik and Scanziani, 2010). This study also found that the ratio between excitation and inhibition that each L5 target neuron received during $\mathrm{L} 2 / 3$ photoactivation correlated well with the impact on its firing rate. Despite this and other work, the 
functional impact of L2/3 activity to sensory responses in L5, particularly in the awake, actively sensing animal, remains uncertain.

We used L2/3-specific optogenetic manipulations in headfixed, whisking mice to address this question. When we optogenetically activated L2/3, many L5 units showed significant suppression, whereas only half as many showed facilitation. Conversely, when we optogenetically deactivated L2/3 during active touch, L5 units were 2 times more likely to be significantly facilitated than suppressed. Intracellular recordings in brain slices revealed that $\mathrm{L} 2 / 3$ potently recruited $\mathrm{L} 5$ somatostatin $\left(\mathrm{SST}^{+}\right.$) interneurons, raising the notion that feedforward, dendritic inhibition may contribute to these effects. Indeed, optogenetically deactivating $\mathrm{SST}^{+}$neurons in vivo significantly altered the functional impact of L2/3 onto L5. Importantly, the net impact of L2/3 activity during active touch was to sharpen the representation of space in individual L5 neurons, both by sparsening their receptive fields and by steepening the slope of activity across tactile space. These experiments demonstrate that the core, descending pathway in the intracortical microcircuit enhances stimulus selectivity and expands the range of cortical output by augmenting the representation of space in the main cortical projection layer.

\section{Materials and Methods}

\section{Experimental model and subject details}

Mice between 6 and 9 weeks of age and of either gender were used for all in vivo experiments. All procedures were approved by the Animal Care and Use Committee of University of California Berkeley. Animals were maintained on a 12:12 reversed light/dark cycle. All DRD3 mice (Tg(Drd3-cre)KI196Gsat/Mmucd) were heterozygous for Cre recombinase and F1 outcrossed with a WT albino mouse line (CD-1, Charles River) to facilitate wheel-running behavior and prevent genetic instability associated with homozygosity in the line. Nine mice with a single recording session each were used for the L2/3 (DRD3) deactivation experiments. Previous in vitro measurements of inhibitory currents indicate that Cre-mediated viral expression of opsins is exclusive to excitatory cortical neurons in this mouse line (Pluta et al., 2015). Three mice with a single recording session each were used for the L2/3 (DRD3) activation experiments. Four mice with a single recording session each were used for the $\mathrm{SST}^{+}$deactivation alone experiments. Three mice with two recording sessions each $(>200 \mu \mathrm{m}$ separation between electrode sites) were used for the two-color SST ${ }^{+}$deactivation and $\mathrm{L} 2 / 3$ activation experiments. SST-cre mice were F1 outcrossed to facilitate wheelrunning and increase fecundity, and therefore were heterozygous for Cre-recombinase.

\section{Methods details}

Neonatal viral injection and in utero electroporation. After the induction of cryogenic anesthesia, neonatal mice (P0-P4) were injected with flexed halorhodopsin 3.0 (AAV9.EF1a.DlO.eNPHR3.0-EYFp.WPRE.hGH) or flexed channelrhodopsin 2 (AAV9.CAGGS.Flex.ChR2-tdTomato.WPRE.SV40). The injection site was targeted to the barrel cortex, at three locations, and at two depths (300 and $150 \mu \mathrm{m}$ ) for each location. At each location and depth, $18.4 \mathrm{nl}$ of virus was injected (Nanoject II) through a beveled glass pipette (WPI, 4878), for a total of $110.4 \mathrm{nl}$ per neonate. In the two-color optogenetic experiments (see Fig. 3), DIO.eNpHR3.0-YFP was injected in SST-Cre mice in the same fashion. For in utero electroporation, pCAG-ChR2-mRuby3 plasmids were injected into the lateral ventricle of E15 embryos, thereby only transfecting L2/3 excitatory neurons (Hakim et al., 2018). The procedure was performed as previously described (Adesnik and Scanziani, 2010).

Preparation for in vivo electrophysiology. Anesthesia was induced with $5 \%$ isoflurane and then maintained at $1.5 \%-3 \%$ during surgery. Respiratory rate and response to toe/tail pinching were monitored throughout surgery to ensure adequate anesthetic depth; $0.05 \mathrm{mg} / \mathrm{kg}$ of buprenorphine was administered for postoperative analgesia. After disinfecting the scalp with $70 \%$ alcohol and $5 \%$ iodine, the skin and fascia above the sensory cortices were removed with surgical instruments. Following application of Vetbond (3M) to the skull surface and wound margins, a custom stainless-steel headplate was fixed to the skull with dental cement (Metabond, Parkell). The headplate was mounted on an angle $(\sim 40$ degrees), to allow a vertical insertion of the electrode to enter the barrel cortex perpendicular to the surface. Two days after surgery, mice were habituated for $4-8 \mathrm{~d}$ to head fixation on a free-spinning circular treadmill, until they freely ran at a fast and steady pace $(>40 \mathrm{~cm} / \mathrm{s})$. In preparation for electrophysiology, mice were briefly (10-15 $\mathrm{min})$ anesthetized with isoflurane, the skull over $S 1$ was thinned with a dental drill (Foredom), and a small $(<200 \mu \mathrm{m})$ craniotomy was made with a 27 gauge needle. In most cases, intrinsic signal imaging was used to localize the $\mathrm{C} 2$ or $\mathrm{C} 1$ barrel column. In all other cases, stereotaxic coordinates were used to localize the macrovibrissae region. Using this approach, sometimes a second craniotomy was made, if an appropriate macrovibrissal column $(\mathrm{C} 2, \mathrm{C} 1, \mathrm{~B} 2, \mathrm{~B} 1)$ was not localized with the first penetration. The small size of the craniotomy minimized motion of the brain during electrode penetration and animal movement. A 16or 32-channel linear silicon probe (NeuroNexus) was guided into the brain using a micromanipulator (Sutter Instruments or Sensapex) and a stereomicroscope (Leica MZ6).

Spike sorting. Sixteen to 32 channels of electrodes spaced in a linear array were amplified (AM Systems) and digitized at $30 \mathrm{kHz}$ (National Instruments) using custom acquisition software (MATLAB, The MathWorks). Spike detection was performed using the UltraMegaSort 2000 package in MATLAB (Hill et al., 2011). After detection, spikes were automatically sorted into clusters of units. Units were then further sorted manually to meet inclusion criteria and prevent pseudo-replication. Quality metrics included analysis of spike amplitude, spike rate, autoand cross-correlation, interspike interval, outlier removal, distance from threshold, and cortical depth of largest waveform. With the exception of a small subset of fast-spiking (FS) or bursting units, included units had no more than $1 \%$ of their individual waveforms violating a refractory period of $2.5 \mathrm{~ms}$.

Spike waveform classification. FS units were separated from regular spiking (RS) units using a $k$-means cluster analysis of two waveform components, as previously published (Pluta et al., 2015, 2017). One component was the normalized amplitude asymmetry between the two positive-going peaks. Positive values indicate that the second peak is larger, whereas negative values indicate the opposite. The other component was the trough-to-peak duration of the large negative-going deflection. FS units were categorized by a larger second positive-going peak (positive amplitude asymmetry), and a short $(<0.33 \mathrm{~ms}$ ) trough-to-peak duration, following previously established approaches (Reyes-Puerta et al., 2015). Units falling into a time bin containing both RS and FS classification were excluded from analysis, as previously published (Pluta et al., 2015, 2017).

In vitro electrophysiology. Brain slices from Drd3-Cre;GIN-GFP 2.5- to 4 -week-old mice were prepared and recorded as previously published (Pluta et al., 2015). ChR2 was expressed in L2/3 using neonatal viral injections, as described above. PCs and $\mathrm{GFP}^{+}$cells in $\mathrm{L} 5$ were patched in the whole-cell configuration. L2/3 was stimulated with a ramp of blue light targeted at an area with a height of $300 \mu \mathrm{m}$ and a width of $600 \mu \mathrm{m}$ ( $\sim 3$ barrel columns) using a digital micromirror device. The spike rate and total synaptic excitatory charge were measuring during the illumination period. Excitatory charge was calculated as the cumulative sum of inward current.

Whisker tracking. A high-speed camera (Basler, acA2000-340kc) was placed below the running wheel, and the full whisker pad was imaged from below using a mirror angled at 45 degrees. A strong infrared light source was hung from above and diffused with thin tissue paper (Kimwipes). High-speed videos were acquired at $500 \mathrm{fps}$ with a $100 \mu \mathrm{s}$ exposure and synchronized via external triggers. The whiskers were traced in MATLAB using custom tracking software (Clack et al., 2012). The mean angle trace of the traced whisker pad was used to calculate the kinematic features during touch: set-point (median angle of envelope) and amplitude (half-width of envelope). 


\section{Experimental design and statistical analysis}

Tactile and optical stimulation. During electrophysiological recording, a vertical $0.7 \mathrm{~mm}$ Hex key (McMaster-Carr) was presented pseudorandomly at one of eight locations (2.2 $\mathrm{mm}$ separation) along the horizontal axis of the whisking field, perpendicular to whisking motion and $\sim 1 \mathrm{~cm}$ away from the mouse's face. For each trial, the Hex key quickly $(<50 \mathrm{~ms})$ entered the whisking field and was held still for $1.5 \mathrm{~s}$ before quickly retracting from the whisking field via a stepper motor (Oriental Motor) under digital control (National Instruments). Between trials, the stepper motor and stimulus pole were automatically moved to the position of the next trial using a motorized linear stage (Zaber). An optical fiber ( $1 \mathrm{~mm}$ core) coupled to an electronically controlled LED (Thorlabs) was positioned directly above the barrel cortex and immersed in saline using a micromanipulator. The thinned region of the skull became invisible/transparent to the eye under saline immersion. Upon hitting the surface of the brain, the light scattered, covering the entire macrovibrissal region. Red $\left(617 \mathrm{~nm}, 8 \mathrm{~mW} / \mathrm{cm}^{2}\right)$ and blue $(470 \mathrm{~nm})$ light was triggered to activate eNpHR3.0 or ChR2, respectively. Red light was delivered as a square pulse, and blue light was delivered as a linear ramp to minimize synchronized activation and prevent the blockage of spiking that can be caused by strong, prolonged membrane depolarization.

Analysis of spiking data. Among all conditions, firing rates were calculated from the window of time corresponding to the optogenetic manipulation period (750 ms for experiments with red light for eNpHR3.0). Evoked firing rates were determined by subtracting the firing rate during the baseline period from the stimulation period, on a trial-by-trial basis.

The optogenetic modulation index (OMI) was computed from the difference in raw firing rates between the light and control conditions, divided by their sum as follows:

$$
O M I=\frac{\text { spikes per } s(\text { light })-\text { spikes per } s(\text { control })}{\text { spikes per } s(\text { light })+\text { spikes pers }(\text { control })}
$$

The sign of optogenetic modulation was computed similarly as the OMI, but based on the number of object positions with an OMI increase $>0.1$ minus the number of object positions with an OMI decrease $<-0.1$, divided by total number of modulated positions (OMI $>0.1$ or $<-0.1$ ). The depth of modulation was calculated as the difference between the largest and smallest sensory responses, divided by their sums.

Spatial selectivity was calculated using a previously published metric referred to as lifetime sparseness (Rolls and Tovee, 1995; Vinje and Gallant, 2000), where the responses, $r$, are the raw firing rate of each neuron divided by the number of stimulus positions, $n$ as follows:

$$
\text { selectivity }=1-\left(\sum_{i=1, n} r_{i} / n\right)^{2} / \sum_{i=1, n}\left(r_{i}^{2} / n\right)
$$

Statistical differences between the control and light groups were determined using standard parametric and nonparametric tests. An $\alpha$ level of 0.05 was used to determine whether a unit significantly changed its firing rate during the optogenetic manipulation period. A two-way ANOVA was used to test for a change in the firing rates between the control and L2/3 deactivation spatial tuning curves, on a unit-by-unit basis. To test for a significant effect during ChR2 activation, a Wilcoxon signed-rank test was performed on the firing rates between the light and spontaneous activity periods of each neuron, on a unit-by-unit basis. Population changes to spatial selectivity indices were tested with a Wilcoxon signedrank test. The surround region of the spatial tuning curves refers to neighboring positions on both sides of the peak, except in the rare instance when the peak was at the first or last position. All statistical measures were performed in MATLAB, using the Statistics toolbox.

Trial inclusion criteria and layer boundaries. Trials containing stimulation periods where the animal's mean run speed dropped $<1.3$ SDs of its population mean were excluded, to ensure consistency in whisking behavior across trials. In addition, trials where the SD of an animal's run speed during stimulation was $>0.8$ SDs from the population mean were excluded. Trials where the animal was simply not running, thresholded by the animal's run speed being $<10 \mathrm{~cm} / \mathrm{s}$, were completely excluded from the analysis of run speed distributions. On average, $16 \pm 1$ trials/ condition were included from each mouse with a minimum treadmill speed of $33.7 \pm 3.2 \mathrm{~cm} / \mathrm{s}$ ( $n=9$ animals, eNpHR3.0 dataset). The depth of each unit was assigned based on the calculated depth of the electrode on the linear array that exhibited its largest waveform. Layer boundaries were confirmed post hoc by labeling of the electrode track with a dye.

\section{Results}

\section{Optogenetic activation of layer 2/3 primarily suppresses L5}

A multielectrode linear array was inserted into the barrel cortex of mice that were habituated to run on a circular treadmill. With their full whisker pad intact, continuously locomoting mice rhythmically and repeatedly touched an object that was momentarily ( $1.5 \mathrm{~s}$ ) placed near the center of the multiunit spatial receptive field, generating reproducible activity trial to trial (Sofroniew et al., 2014; Pluta et al., 2015, 2017). Neural activity was semiautomatically sorted into individual units and classified as pyramidal/RS or putative FS interneurons as previously published (Pluta et al., 2015, 2017; Reyes-Puerta et al., 2015). To test the functional impact of L2/3 onto L5, we first selectively expressed an optogenetic activator, ChR2, in L2/3 excitatory neurons using a Credependent AAV vector in a L2/3-excitatory neuron-specific Cre-specific driver line (DRD3-Cre; Fig. 1) (Pluta et al., 2015). By systematically varying the intensity of light emitted from an optical fiber ( $1 \mathrm{~mm}$ core) placed directly above $(<1.5 \mathrm{~mm})$ the barrel cortex, we were able to generate mean firing rates in $\mathrm{L} 2 / 3$ similar to those generated by active touch (Fig. $1 A, B$ ). Nearly all ( 18 of 19) recorded L2/3 excitatory neurons increased their firing rate above baseline during the light period (positive OMI; see Materials and Methods), confirming the efficacy of the optogenetic manipulation (Fig. $1 C ; p<0.001$, Wilcoxon signed-rank

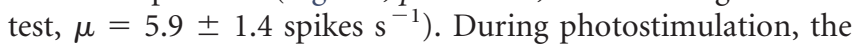
trial-to-trial variability of $\mathrm{L} 2 / 3$ firing rate was not consistently different from that evoked by sensory stimulation, as quantified by the Fano factor for each isolated unit ( $p=0.47, n=19$, Wilcoxon signed-rank test).

Next, we asked whether direct optogenetic drive of L2/3 would enhance, suppress, or have no impact on the spontaneous activity of L5. Optogenetically activating L2/3 significantly modulated the spontaneous firing rate in the majority of L5 units $(62 \%$, Wilcoxon signed-rank test, $\alpha=0.05$ ). Three example units illustrate the effects of touch and L2/3 activation on L5 RS units (Fig. $1 D)$. In two of these units, the impact of touch and the impact of L2/3 activation onto L5 activity were remarkably similar. Overall, $41 \%$ of L5 RS units had their spontaneous activity significantly suppressed by activating L2/3, whereas only half as many (21\%) were significantly facilitated (Wilcoxon signed-rank test, $\alpha<$ 0.05 ; Fig. $1 E$, pie charts). Notably, practically all of the L5 neurons that were suppressed by touch were also suppressed by activating $\mathrm{L} 2 / 3$, implying that intracortical processing is integral for touch suppression in L5. A minority of L5 RS units were touchfacilitated at their receptive field center but suppressed by L2/3 activation (Fig. $1 D$, middle, $F$, bottom right). Across the population, the impact of touch and the impact of L2/3 activation were significantly correlated (Fig. $1 F ; p<0.001, R^{2}=0.53$, multiple linear regression).

\section{Optogenetic deactivation of L2/3 primarily facilitates L5}

Optogenetic activation with ChR2 demonstrates that $\mathrm{L} 2 / 3$ is sufficient to significantly modulate L5, primarily through suppression. However, it is critical to test whether endogenous L2/3 activity would have a similar effect by optogenetically deactivating $\mathrm{L} 2 / 3$ activation during sensory stimulation. We deactivated L2/3 excitatory neurons using the optogenetic silencer, 
A

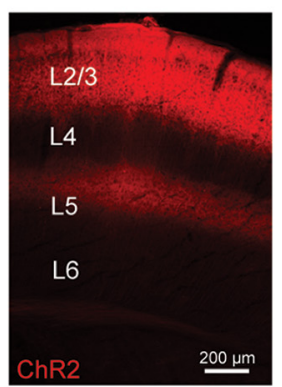

D

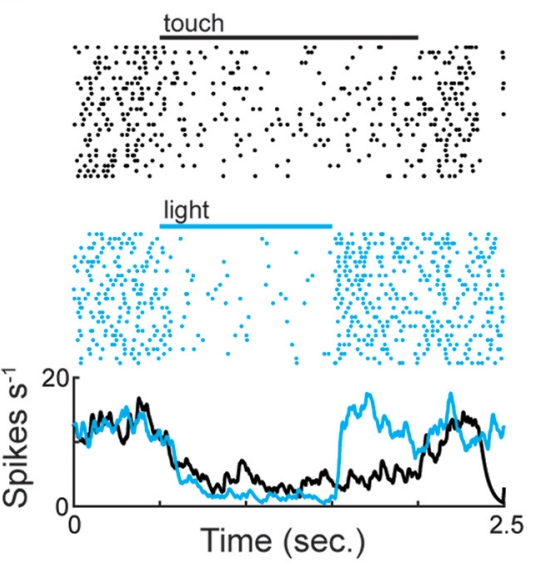

\section{Layer $2 / 3$ data}

B

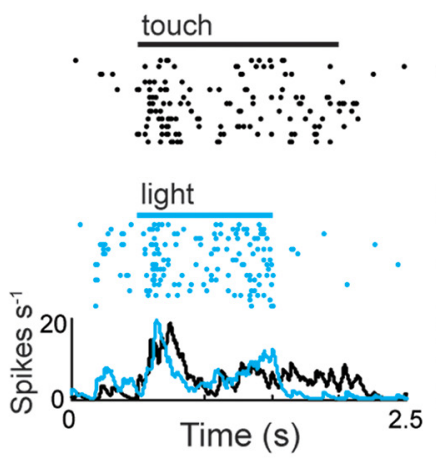

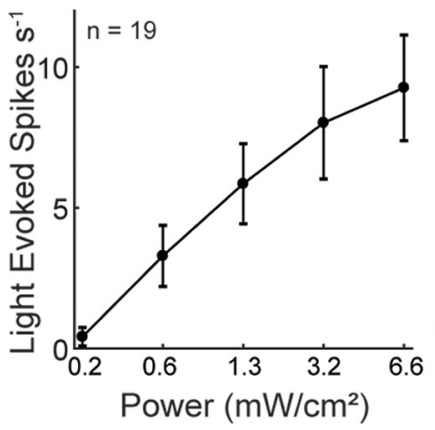

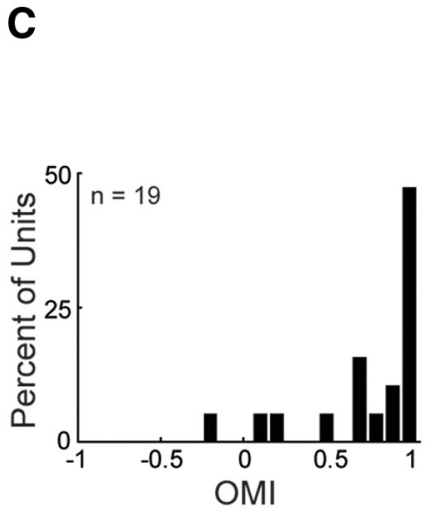

E
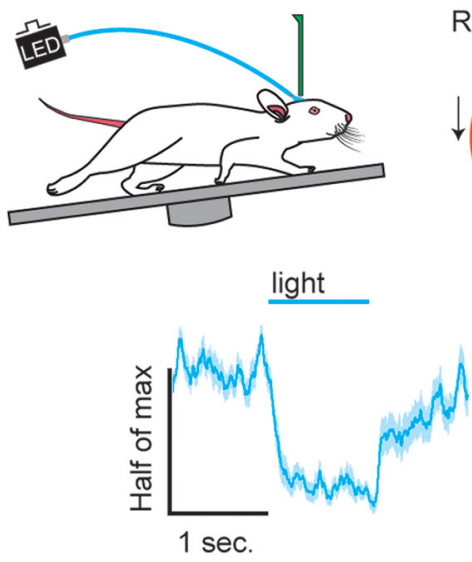
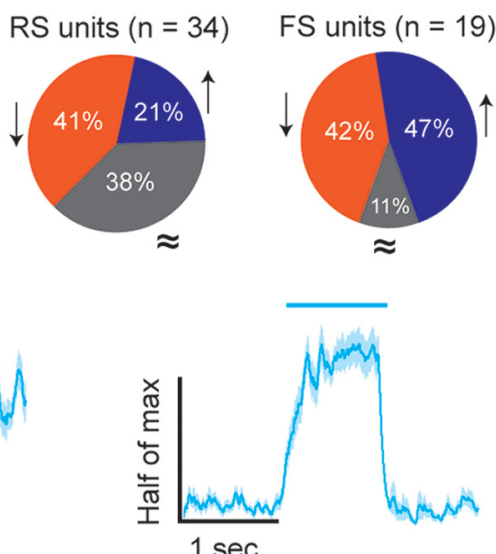

FS units $(n=19)$

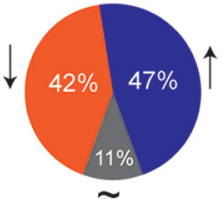

Layer 5 data
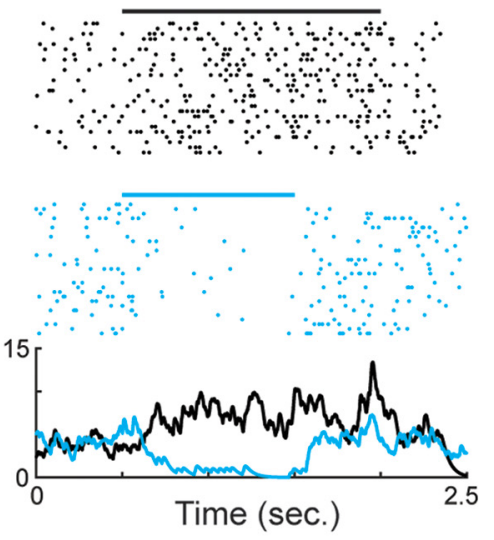

Time (sec.)

\section{F}

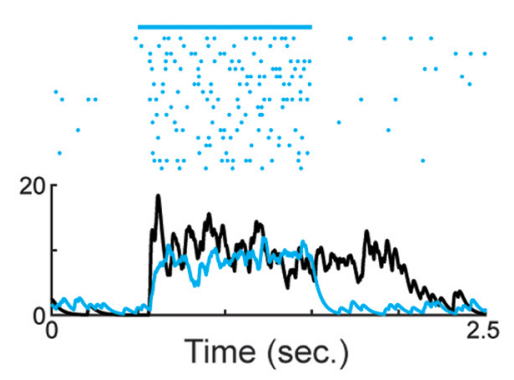

F

C

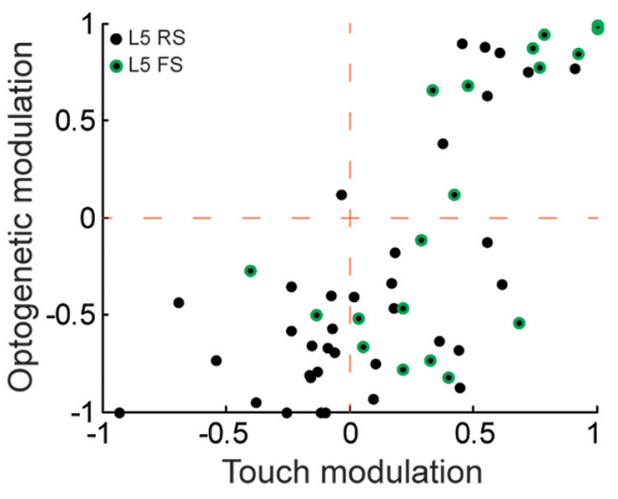

Figure 1. Optogenetic activation of $L 2 / 3$ predominantly suppresses $L 5 . A, A$ histological transverse section through the barrel cortex showing $L 2 / 3$ ChR2 expression with the tdTomato fluorophore. A putative ChR2-expressing layer 2/3 RS neuron that is driven during active touch and activated by a linear ramp of blue light alone. $B$, ChR2 activation dose-response curve showing the relationship between peak light power and firing rate. $L 5$ data were analyzed using $1.3 \mathrm{~mW} / \mathrm{cm}^{2}$ power. $C, 0 \mathrm{MI}$ for the population of $L 2 / 3 \mathrm{RS}$ neurons ( $p<0.001$, Wilcoxon signed-rank test, $n=19$ ). $\boldsymbol{D}$, Three example L5 RS neurons that were suppressed by touch or light (left), facilitated by touch but suppressed by light (middle), or facilitated by touch or light (right). $\boldsymbol{E}$, Top, The percentage of $L 5$ units that were significantly facilitated (blue), suppressed (orange), or unchanged (gray) by $L 2 / 3$ activation (Wilcoxon signed-rank test, $\alpha=0.05$ ). Bottom, Normalized population firing rates of $L 5$ ( $R S$ and $F S$ ) units grouped by the sign of the significant effect. Neurons suppressed by light were normalized to the baseline period (left), whereas neurons facilitated by light were normalized to the light period (right). $\boldsymbol{F}$, Relationship between touch modulation and optogenetic modulation in $L 5$ units $\left(p<0.001, R^{2}=0.53, n=55\right.$, multiple linear regression). Error bars are the standard error of the mean.

eNpHR3.0, in the same L2/3 Cre-driver line (Fig. 2). We mapped neuronal receptive fields by placing a vertical bar pseudorandomly in eight different horizontal locations inside the whisking field, identical to our previous approaches (Pluta et al., 2015, 2017). As we have described previously, in each pole position, the mouse actively contacts the stimulus bar with 2-4 neighboring whiskers. We deliberately chose to keep all whiskers intact, even though this makes identifying individual whisker contacts impractical because it better resembles natural conditions where mice typically contact objects with multiple whiskers on each whisk cycle. Our prior experiments in this paradigm demonstrated that the sensory responses of S1 neurons for each pole 


\section{Layer $2 / 3$ data}

A

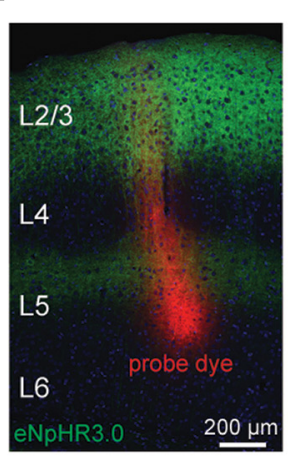

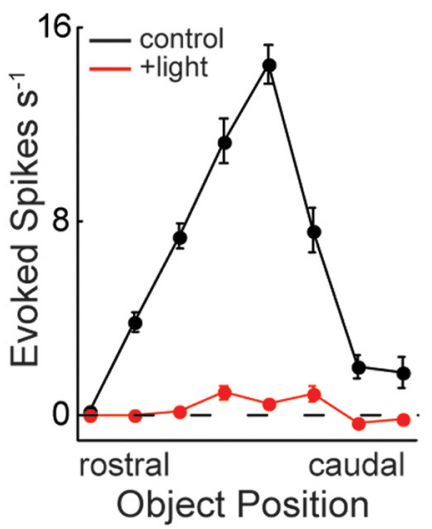
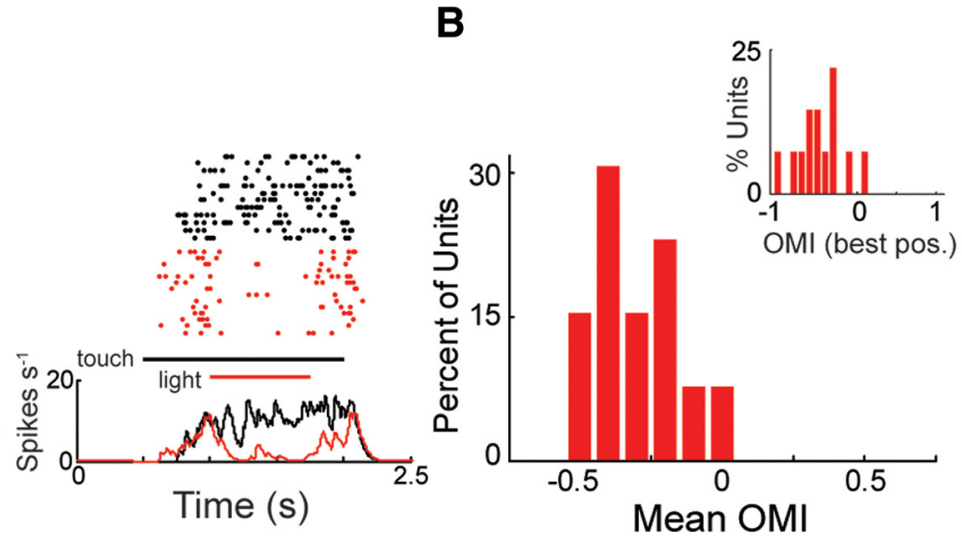

C
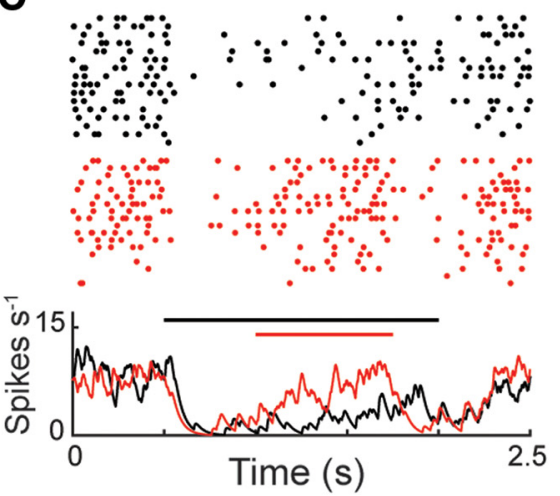

D

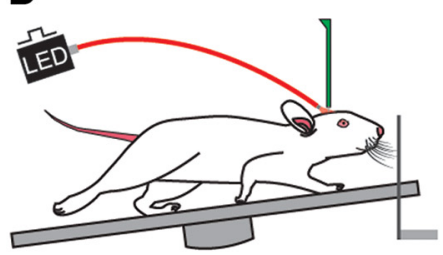

RS units $(n=79)$

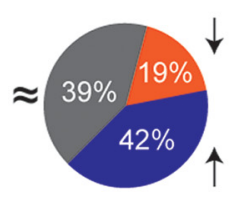

Layer 5 data


E
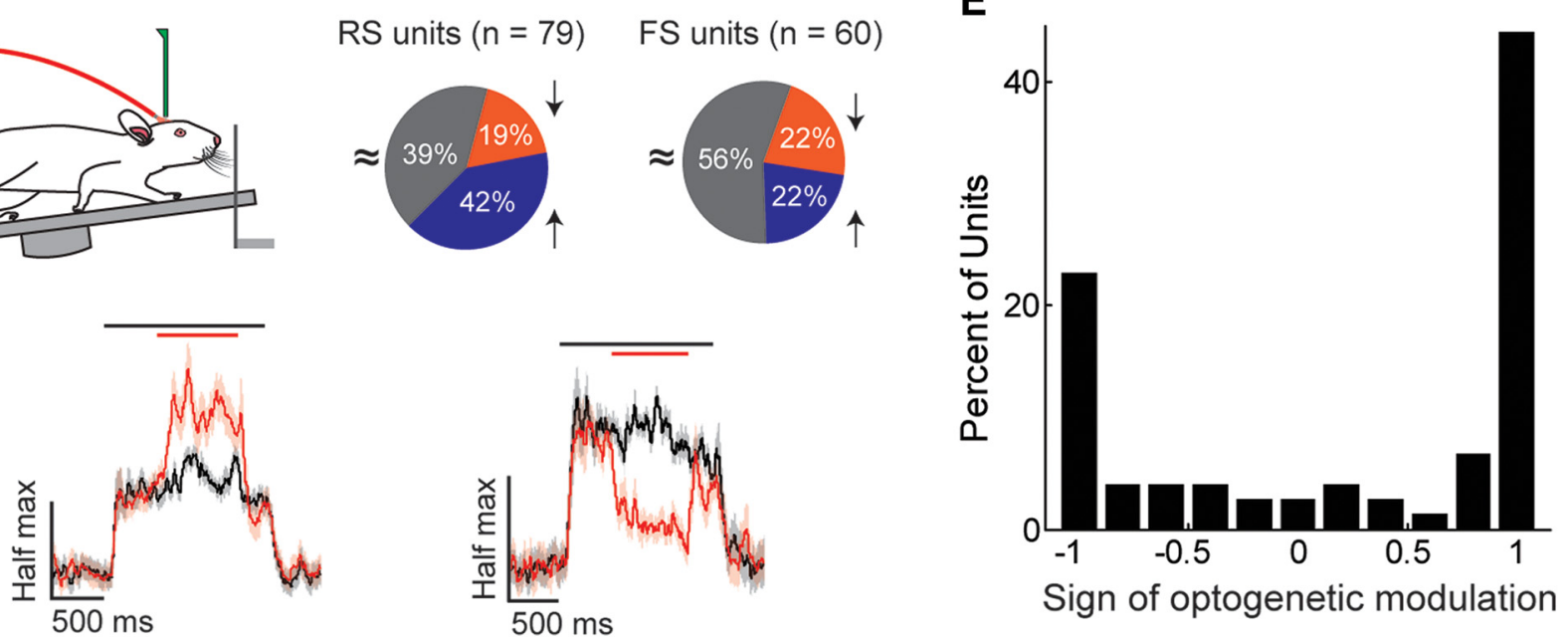

Sign of optogenetic modulation

Figure 2. L2/3 primarily suppresses touch responses in L5.A, A histological transverse section through the barrel cortex showing L2/3 expression of eNpHR3.0 with a green fluorophore. Example receptive field, raster, and peristimulus time histogram showing the effect of red light on a L2/3 neuron putatively expressing eNpHR3.0. B, Mean OMI averaged across the 8 object positions for the L2/3 RS population. Inset, Histogram of OMI for the best stimulus position. C, The impact of L2/3 deactivation on touch-evoked activity in three different L5 units (right). $\boldsymbol{D}$, Top, The percentage of L5 units where the response to active touch significantly increased (blue), decreased (orange), or did not change (gray) during L2/3 deactivation (two-way ANOVA, $\alpha=0.05$ ). Bottom, Normalized population histograms of $L 5$ (RS and FS) firing rates grouped by the sign of the significant effect. The firing rates of each neuron were normalized to their largest control touch response. $E$, Histogram of the sign of optogenetic modulation in L5. Error bars are the standard error of the mean.

position strongly depend on the summed activation of multiple whiskers (Pluta et al., 2017). Numerous studies in the anesthetized rodent have demonstrated that multiple different whiskers drive L5 neurons (Ito, 1992; Ghazanfar and Nicolelis, 1999; Shimegi et al., 1999; Manns et al., 2004; Ego-Stengel et al., 2005; Ramirez et al., 2014). Therefore, multiwhisker touch is necessary for understanding the receptive field properties of L5. Nonetheless, information on the exact pattern of contacts across the whisker field could enable an understanding of cortical dynamics that is unfeasible using the current approach.

Red light illumination $\left(617 \mathrm{~nm}, 1 \mathrm{~mm}\right.$ fiber, $\left.8 \mathrm{mw} / \mathrm{cm}^{2}\right)$ of the entire barrel cortex produced robust suppression of touch re- 
sponses in nearly all recorded L2/3 RS units across their entire receptive fields, confirming the efficacy of $\mathrm{L} 2 / 3$ optogenetic suppression (Fig. $2 A, B ;-63 \pm 10 \%$ change in mean firing rate, $n=$ $13, p<0.01$, Wilcoxon signed-rank test). Three example L5 RS units (Fig. 2C) illustrate the impact of L2/3 deactivation on touch suppression (left) and touch facilitation (middle, right). Overall, the touch response in a small fraction $(19 \pm 7 \%, 15$ of 79 overall, 9 mice) of L5 RS units significantly decreased, whereas in double the fraction of L5 RS units ( $40 \pm 8 \%, 33$ of 79 overall, 9 mice), it significantly increased (two-way ANOVA, $\alpha=0.05$; Fig. $2 D$, top row). Overall, L2/3 significantly modulated touch responses in a substantial fraction $(61 \%, 48$ of 79$)$ of L5 output neurons.

Next, we sought to determine the strength of translaminar modulation in the subset of L5 units that significantly changed their firing rate during L2/3 deactivation (two-way ANOVA, $\alpha=$ $0.05)$. Averaging firing rates independently for significantly facilitated/suppressed units demonstrates that their optogenetic modulation was confined to the light period (Fig. $2 D$, bottom row, red line). Among all L5 units displaying a significant increase in spiking $(n=45)$, firing rates changed by $30 \pm 10 \%$ at the receptive field center (Fig. $2 D$ ). Among all L5 units showing a significant decrease in spiking $(n=28)$, firing rates changed by $-36 \pm 3.5 \%$ at the receptive field center (Fig. $2 D$ ). Therefore, $\mathrm{L} 2 / 3$ has a substantial impact on touch responses in L5.

To describe the consistency of L2/3's impact on each L5 unit, we calculated an index that describes the sign of optogenetic modulation across their receptive field (see Materials and Methods). A positive index indicates that the firing rate increased during $\mathrm{L} 2 / 3$ deactivation at more object positions than it decreased, whereas a negative index indicates the opposite. Most (50 of 73, $68 \%$ ) L5 units had an index of either -1 or 1 , indicating that, in these units, the sign of L2/3's effect onto L5 was consistent across tactile space (Fig. 2E).

Because optogenetic manipulation of L2/3 could alter the whisking pattern of mice, we tracked the whiskers with a highspeed camera in a subset of mice and found that optogenetic manipulations of $\mathrm{L} 2 / 3$ had no impact on the amplitude or setpoint of the whisking envelope (Fig. $3 A-C$ ). In addition, the variation in whisker kinematics was similar between the control and light conditions, indicating that the reliability of whisking was unaffected by L2/3 deactivation. We have previously shown that illuminating the brain of mice under identical experimental conditions while expressing a fluorescent protein without an optogenetic actuator has no effect on the activity of the somatosensory cortex, indicating that light alone does not impact cortical responses in S1 (Pluta et al., 2015). To understand whether multiwhisker touch was necessary for the effects we observed, in 3 separate mice, we optogenetically deactivated L2/3 (DRD3eNpHR3.0) during touch with only the principal whisker intact (Fig. $3 D, E$ ). Similar to our main dataset with the full whisker pad intact, the optogenetic modulation of L5 RS units was primarily positive ( $p<0.001, n=41$, Wilcoxon signed-rank test), whereas activity changes across the L5 FS population were evenly mixed with no consistent effect ( $p=0.81, n=19$, Wilcoxon signedrank test).

\section{L2/3 recruits SST interneurons to suppress L5 output}

What circuits enable L2/3 to suppress touch responses in L5? One possibility is that feedforward inhibition from L2/3 onto L5 plays a crucial role. Previous work in brain slices demonstrated that activation of L2/3 generates a mixture of excitation and inhibition in L5 PCs, yet the source of this inhibition remains unknown (Adesnik and Scanziani, 2010). L2/3 neurons monosynaptically innervate both parvalbumin $\left(\mathrm{PV}^{+}\right)$neurons and SST ${ }^{+}$neurons in L2/3 and L5, which themselves can synapse onto L5 somata and dendrites, respectively (Reyes et al., 1998; Xiang et al., 2002; Markram et al., 2004; Otsuka and Kawaguchi, 2009; Apicella et al., 2012; Jiang et al., 2015; Naka and Adesnik, 2016). Because GABAergic $\mathrm{PV}^{+}$neurons can often be putatively identified in vivo by their short, FS waveform, we first determined the impact of optogenetic manipulations of L2/3 on L5 FS units in vivo (Celio, 1986; Kawaguchi et al., 1987). It is important to note that $\sim 77 \%$ of units identified as FS using extracellular waveforms are $\mathrm{PV}^{+}$, with the remaining probably corresponding to FS PCs or other types of GABAergic neurons (Moore and Wehr, 2013; Muñoz et al., 2014). If FS units are the sole contributors of translaminar inhibition from L2/3 to L5, then one would expect that most of the FS units to be facilitated by $\mathrm{L} 2 / 3$, as is the case when optogenetically manipulating L4 (Pluta et al., 2015). However, optogenetic manipulation of $\mathrm{L} 2 / 3$ was equally likely to suppress or facilitate L5 FS unit activity (Figs. $1 E, 2 D$ ). In addition, the median number of spikes added to or subtracted from an FS neuron's touch response during L2/3 deactivation was equivalent for both groups ( $p=0.33, n=13$ for both, Wilcoxon ranked-sum test). Therefore, we next asked whether $\mathrm{SST}^{+}$neurons might mediate feedforward inhibition onto L5 PCs, as has been shown in brain slices from the motor cortex (Apicella et al., 2012). Because $\mathrm{SST}^{+}$neurons cannot be readily identified by their spike waveforms in vivo, we asked, in vitro, where they can be positively identified in a specific reporter line, GIN (Oliva et al., 2000) and what the impact of $\mathrm{L} 2 / 3$ activation would be on their firing rates. We made intracellular recordings from $\mathrm{L}_{5 \mathrm{GIN}}{ }^{+}$cells while photoactivating L2/3 with blue light. We found that these $\mathrm{SST}^{+}$neurons were consistently driven to high firing rates, which was explained by strong net synaptic excitatory input (Fig. 4). On the other hand, L5 PCs were never driven by photoactivation of L2/3, and approximately half ( 9 of 16) of putative FS interneurons were well driven. Together with the well-established fact that L2/3 can also recruit $\mathrm{SST}^{+}$neurons in $\mathrm{L} 2 / 3$, which may synapse directly onto L5 PC dendrites (Kapfer et al., 2007), these data suggest that $\mathrm{SST}^{+}$neurons, in vivo, could influence the functional relationship between L2/3 and L5 PCs.

To test whether $\mathrm{SST}^{+}$neurons are critical to the response properties of L5 during active sensation, we deactivated them in vivo using eNpHR3.0 in SST-Cre mice. Deactivating SST ${ }^{+}$cells significantly enhanced the firing rates of many L5 RS units during active touch ( 18 of $45,40 \%$, two-way ANOVA, $\alpha=0.05$ ), both by reducing/eliminating touch suppression and by enhancing touch facilitation, consistent with a key role in contributing to the sign and gain of touch responses in L5 (Fig. 5). Nevertheless, this experiment does not directly test the role of SST ${ }^{+}$neurons in the L2/3 to L5 circuit and potentially has off-target effects related to the disinhibition of touch responses in layers $2-4$. To address these issues, we asked whether optogenetically deactivating SST ${ }^{+}$ cells would reduce the ability of $\mathrm{L} 2 / 3$ photostimulation to suppress L5 and enhance the ability of L2/3 photostimulation to facilitate L5. To gain independent optogenetic control of L2/3 neurons in $\mathrm{SST}^{+}$Cre-driver mice, we expressed ChR2 selectively in L2/3 PCs using in utero electroporation (Hakim et al., 2018), and eNpHR3.0 in SST ${ }^{+}$cells by neonatally injecting DIO virus as above (Fig. 6A). As a control for this alternative method of expressing ChR2 in L2/3, we found that optogenetic activation of L2/3 primarily suppressed L5 RS unit activity below baseline when their firing rates were averaged across the entire photostimulation period (49\% suppressed and 26\% facilitated, Wilcoxon signed-rank test, $\alpha=0.05$ ), largely agreeing with our viral 
A

Whisker tracking analysis

B


D
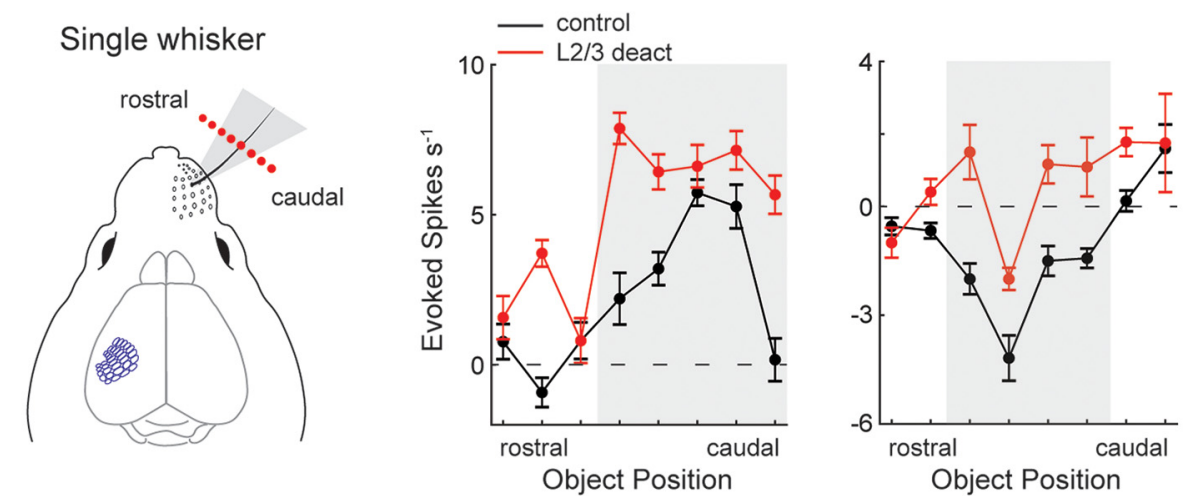

E



Figure 3. L2/3 deactivation does not change the set-point or amplitude of whisking during touch. $A$, An image from the processed whisker tracking video showing the tracing of the whiskers (magenta) and their mean position (green). $\boldsymbol{B}$, Left, Diagram illustrating how whisker set-point was calculated, which was defined as the median angle of the whisking envelope. Right, Comparison of whisker set-point during touch alone (black) and touch combined with L2/3 deactivation (red) across 3 mice. C, Left, Diagram illustrating how the amplitude of whisker motion was calculated, which was defined as the half-distance between the top and bottom of the whisking envelope. Right, Comparison of whisk amplitude during touch alone (black) and touch combined with L2/3 deactivation (red) across the same 3 mice. In some mice, a brief $(\sim 200 \mathrm{~ms})$ increase in whisk amplitude occurred at the onset of object presentation but ended before $L 2 / 3$ deactivation. $L 2 / 3$ deactivation had no effect on the amplitude or set-point of whisking in any of the mice. All line plots indicate mean $\pm 95 \%$ Cl.D, Diagram of the experiment with only the principal whisker intact. With the principal whisker alone, touch occurred only at a subset of object positions (gray). Two example L5RSneurons show the facilitation of touch responses during L2/3 deactivation (DRD3-NpHR3.0). E, Mean optogeneticmodulation of activity in the principal whisker contact zonefor the population of $\mathrm{RS}(n=41)$ and FS $(n=19)$ cells from 3 mice. Error in the tuning curves are the standard error of the mean.

expression results using the $\mathrm{L} 2 / 3$ Cre-driver mice. In the suppressed units, we asked whether there was an initial period of facilitation at the very onset of photostimulation, possibly consistent with the reported slow onset of $\mathrm{SST}^{+}$recruitment; however, there was no such effect.
An example putative $\mathrm{SST}^{+}$neuron, identified by its complete suppression during red light, was potently activated during $\mathrm{L} 2 / 3$ photostimulation, implying that $\mathrm{L} 2 / 3$ is capable of strongly facilitating $\mathrm{L}_{5} \mathrm{SST}^{+}$neurons in vivo (Fig. $6 B$ ). If SST ${ }^{+}$neurons are critical to the connection between $\mathrm{L} 2 / 3$ and $\mathrm{L} 5$, then $\mathrm{SST}^{+}$deac- 
A

L2/3 photo-activation whole-cell: L5 PC, GIN+ or FS

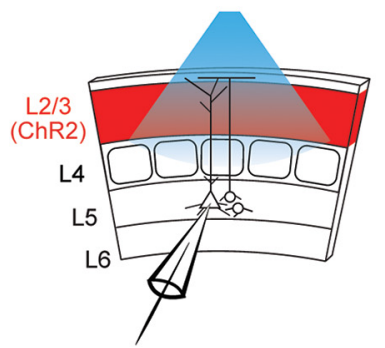

B

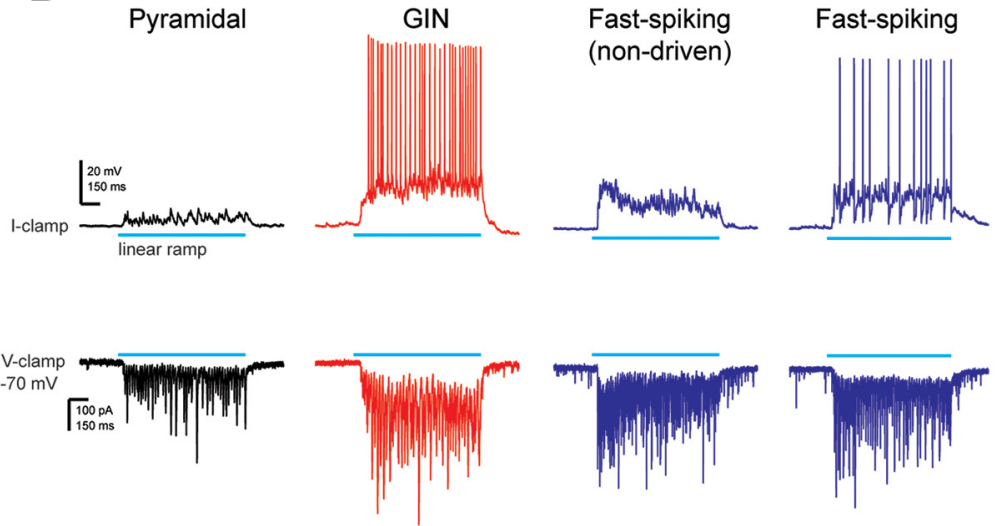

C

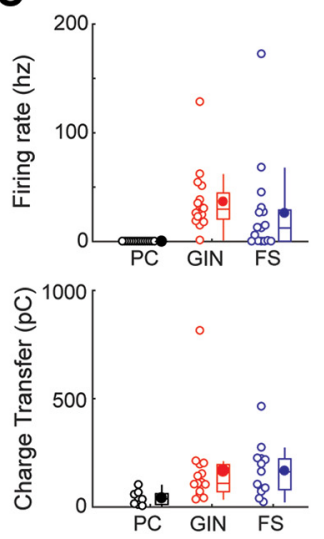

Figure 4. $L 2 / 3$ drives $\mathrm{LSSST}^{+}$interneurons. $A$, Diagram of the experimental setup. Intracellular recordings were performed on $\mathrm{L} 5 \mathrm{PC}$, genetically labeled $\left(\mathrm{GIN}^{+}\right) \mathrm{L} 5 \mathrm{SST} \mathrm{T}^{+}$, or FS interneurons, whereas L2/3 was activated using ChR2 that was virally expressed using the DRD3-Cre driver. $\boldsymbol{B}$, Top row, Membrane potential from an example L5 PC (black), a L5SST ${ }^{+}$cell (red), and two L5 FS cells (blue) during L2/3 activation with a linear ramp of light (blue bar, $1 \mathrm{~s}$ duration). Bottom row, Excitatory currents from these same neurons during L2/3 activation with a linear ramp of light. Voltage was held at $-70 \mathrm{mV}$. C, Comparison of firing rate distributions for these groups of cell types. Bottom, Comparison of excitatory charge transfer between these groups.

A
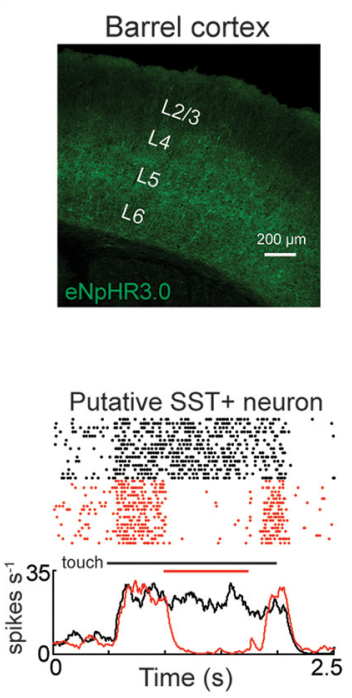

B
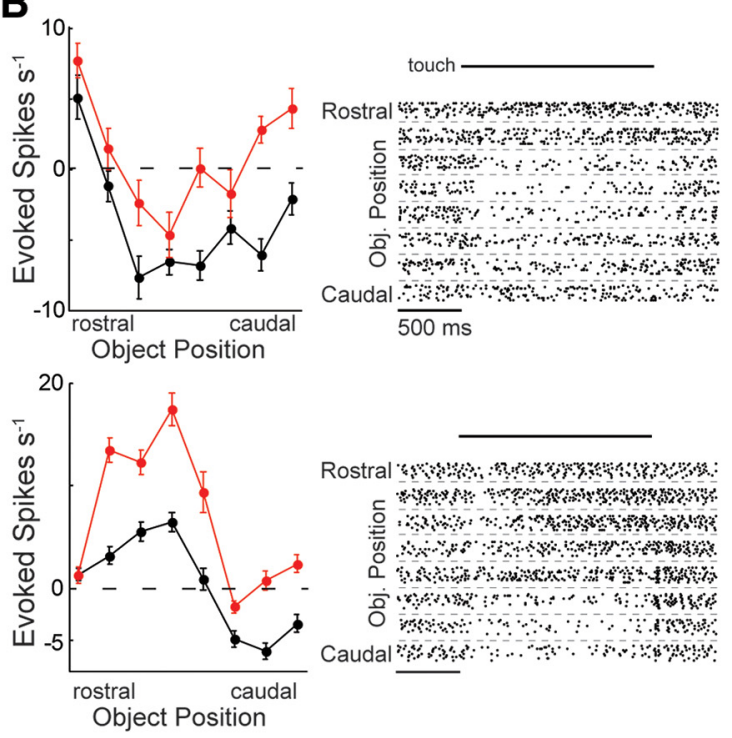

C

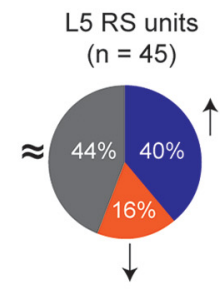

L5 FS units $(n=22)$
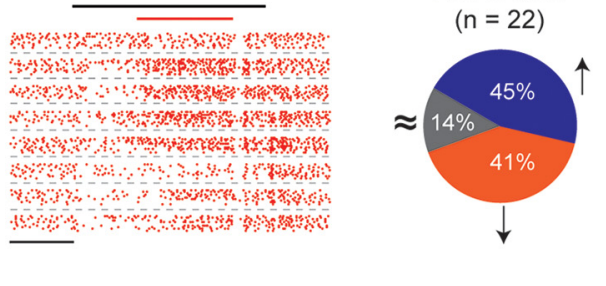

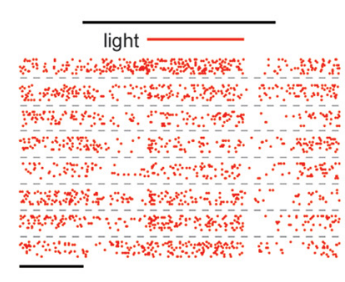

Rostral 4 mon : - When 응 o 0 sing 迹 O Caudal

Figure 5. SST ${ }^{+}$deactivation facilitates touch responses in L5RS units. $\boldsymbol{A}$, Top, Histological transverse section from an SST-cre mouse expressing eNpHR3.0 tagged with a green fluorophore (YFP). Bottom, Putative SST ${ }^{+}$neuron from this mouse that had its activity nearly abolished during the light period. $\boldsymbol{B}$, Tuning curves and raster plots from two example L5 RS neurons that significantly increase their firing rate during SST ${ }^{+}$deactivation (two-way ANOVA, $\alpha=0.05$ ). In the raster plots, only the first 10 trials are shown for each condition to simplify visualization. $C$, The fraction of L5 neurons that significantly go up (blue), go down (orange), or do not change (gray) during SST ${ }^{+}$deactivation (two-way ANOVA, $\alpha=0.05$ ). Error bars are the standard error of the mean.

tivation combined with L2/3 activation should disinhibit L5 more than $\mathrm{SST}^{+}$deactivation does alone. Indeed, as shown in two example L5 RS units, $\mathrm{SST}^{+}$inactivation alone (no touch, no blue light) had no significant effect, whereas activating L2/3 alone caused suppression (top) or facilitation (bottom) (Fig. 6C). However, when we superimposed $\mathrm{SST}^{+}$deactivation onto $\mathrm{L} 2 / 3$ activation, we observed a prominent disinhibitory effect; and in some cases, L2/3-mediated suppression was converted into facilitation (Fig. 6C, top, $D$, top left).

Across the population of L5 putative PCs, $\mathrm{SST}^{+}$deactivation significantly reduced the suppressive effect and enhanced the facilitative effect of L2/3 activation ( $p<0.001, n=91$, Wilcoxon signed-rank test; Fig. $6 D$ ). Furthermore, the disinhibitory effects (positive OMI) of SST ${ }^{+}$deactivation were significantly enhanced by photoactivating L2/3 ( $p<0.001, n=91$, Wilcoxon signedrank test; Fig. $6 E$ ). A population-averaged histogram of normalized firing rates demonstrates the greater disinhibitory effect of
$\mathrm{SST}^{+}$deactivation when combined with L2/3 activation (Fig. $6 F)$. This greater disinhibitory effect was also apparent on raw firing rates ( $p<0.001, n=91$, Wilcoxon signed-rank test). Furthermore, this increase in firing rate from $\mathrm{SST}^{+}$deactivation combined with $\mathrm{L} 2 / 3$ photoactivation was significantly greater than the linear sum of each component optogenetic response ( $p=0.007, n=91$, Wilcoxon signed-rank test). Together, these data argue that $\mathrm{L} 2 / 3$ recruits $\mathrm{SST}^{+}$inhibition to augment translaminar suppression and moderate translaminar facilitation in L5 PCs.

\section{L2/3 enhances the selectivity and range of L5 output}

We next sought to address the net impact of L2/3 activity on sensory coding in L5. To address this question, we analyzed how L5 encodes actively scanned space (Fig. 7A). Previous work in the barrel cortex and other sensory areas has shown that sensory responses in L5 neurons are heterogeneous, and show a mix of 
A

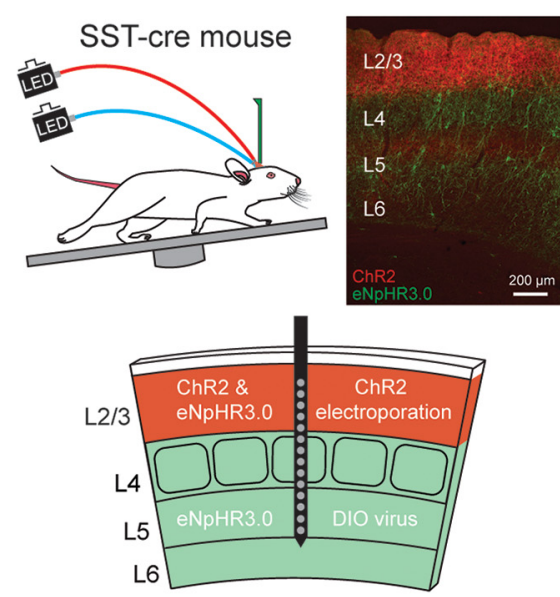

B

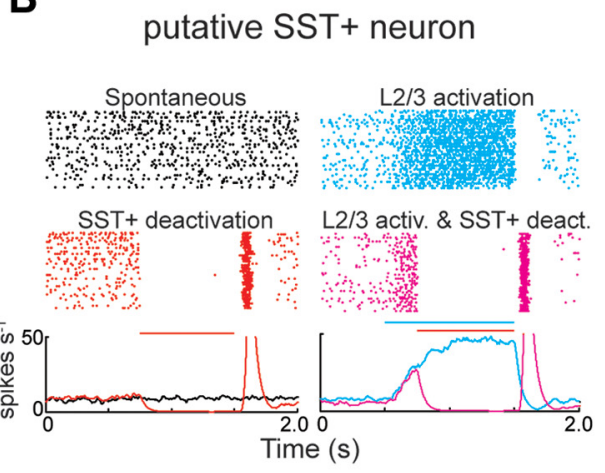

C

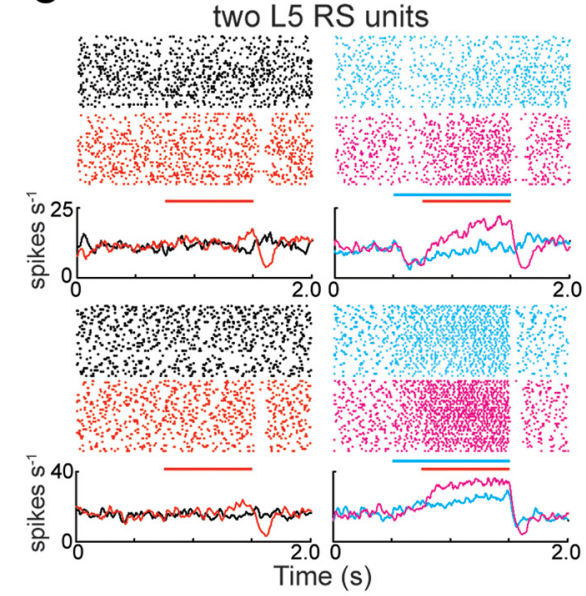

two L5 RS units

D

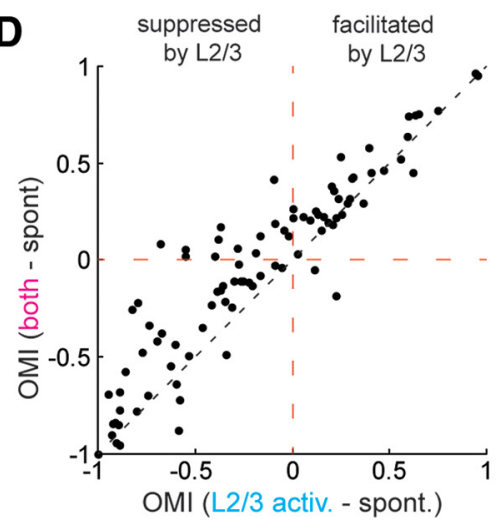

E

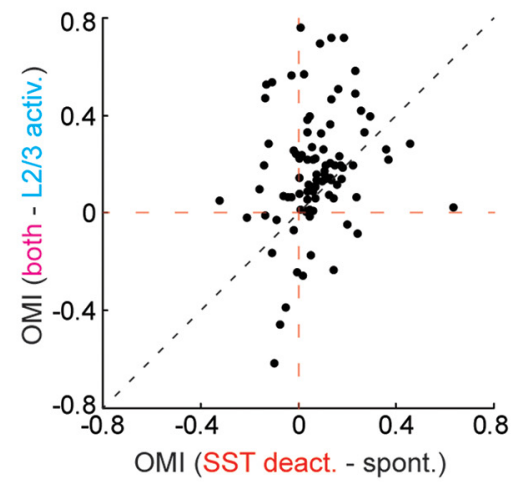

$\mathbf{F}$

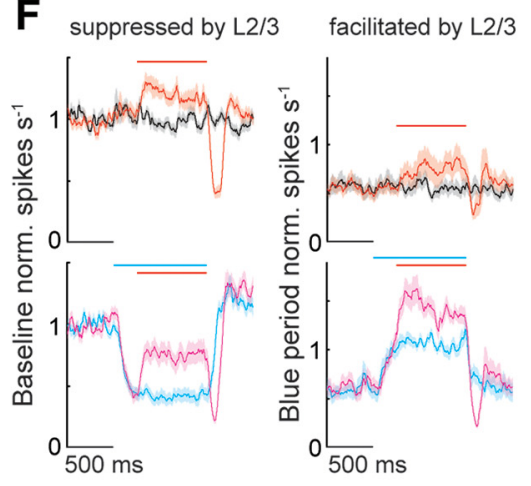

Figure 6. $\mathrm{L} 2 / 3^{\prime}$ 's functional impact onto $\mathrm{L} 5$ is partially mediated by SST ${ }^{+}$interneurons. $\boldsymbol{A}$, A histological transverse section through the barrel cortex showing ChR2 expression in tdTomato and eNpHR3.0 expression in YFP. DI0-eNpHR3.0 was virally expressed in all layers, whereas ChR2 was expressed only in L2/3 using in utero electroporation. $B$, Spike raster of a putative SST ${ }^{+} \mathrm{L} 5$ interneuron during spontaneous periods (black), during L2/3 photoactivation (blue), during SST ${ }^{+}$photodeactivation (red), and during both presented simultaneously (magenta). $\boldsymbol{C}$, As in $\boldsymbol{B}$, but for two example L5 RS units where optogenetic deactivation of SST ${ }^{+}$neurons removed L2/3-mediated suppression (top) or enhanced L2/3-mediated facilitation (bottom). $\boldsymbol{D}, 0 \mathrm{Ml}$ illustrating the effect of $\mathrm{L} 2 / 3$ activation on Spontaneous activity versus the effect of $\mathrm{L} 2 / 3$ activation combined with SST ${ }^{+}$deactivation on spontaneous activity. $\boldsymbol{E}, 0 \mathrm{Ml}$ scatter plot illustrating the greater disinhibitory effect of SST ${ }^{+}$deactivation, whereas $\mathrm{L} 2 / 3$ is photoactivated $(p<$ $0.001, n=91$, Wilcoxon signed-rank test). $F$, peristimulus time histograms of mean \pm SEM of $L 5 \mathrm{RS}$ unit firing rates during spontaneous activity and the three forms of optogenetic stimulation. Neurons were grouped by the sign of the effect of $L 2 / 3$ activation and normalized as indicated. Error bars are the standard error of the mean.

facilitation and suppression (Krupa et al., 2004; Curtis and Kleinfeld, 2009; de Kock and Sakmann, 2009; O’Connor et al., 2010; Sofroniew et al., 2015; Qi et al., 2016; Takahashi et al., 2016). Because spontaneous activity in L5 is uniquely high for cortical neurons, it is able to encode sensory stimuli with a decrease in activity (de Kock and Sakmann, 2009). Consistent with these data, we found that the L5 RS unit population displayed a heterogeneous mixture of touch facilitation and suppression across their receptive fields. While some L5 RS units increased their firing rate for all object locations in their whisking field, most units exhibited rate increases for some object locations and decreases for others, which putatively sharpen their representation of space. Because our optogenetic data above show that L2/3 primarily suppresses L5, we hypothesized that L2/3 sharpens spatial tuning in L5.

Most L5 RS units were significantly tuned to object position (73\%, 58 of 79, one-way ANOVA), whether they were predominantly touch-facilitated or touch-suppressed. In most units, demonstrated in two example neurons, L2/3 deactivation elevated firing rates, often reducing touch suppression (Fig. $7 B$ ). To determine the effect of $\mathrm{L} 2 / 3$ deactivation on the spatial selectivity of each unit, we used a metric that quantifies the uniqueness of the peak in a tuning curve (Rolls and Tovee, 1995; Vinje and
Gallant, 2000; Rolls, 2016). Across the population, spatial selectivity significantly decreased during $\mathrm{L}-2 / 3$ deactivation $(p=$ 0.001 , Wilcoxon signed-rank test, $n=58$; Fig. $7 C$, top). The majority of decreases in spatial selectivity were related to an increase in firing rate (Fig. $7 C$, bottom), with a significantly greater increase in the surround than at the peak of the tuning curve ( $p=$ $0.004, n=41$, Wilcoxon signed-rank test, $1.24 \pm 0.44$ more spikes $\mathrm{s}^{-1}$ ). In a small minority of units, spatial selectivity decreased due to a decrease in touch facilitation. Thus, descending intracortical input increases stimulus selectivity in L5 primarily by providing net lateral inhibition. Overall, these data argue that L2/3 activity functions to accentuate the center of L5 receptive fields.

Many L5 neurons possess high baseline firing rates (de Kock and Sakmann, 2008). To better assess stimulus selectivity in these neurons, we calculated their slope of activity across tactile space (Bala et al., 2003; Seriès et al., 2004; Butts and Goldman, 2006). In an example L5 neuron (Fig. 7D), activity at the trough of the receptive field preferentially increased during $\mathrm{L} 2 / 3$ deactivation. To calculate the slope of activity during control stimulation, firing rates for each unit were sorted into descending order and fit with a simple linear regression (Fig. $7 E$ ). Activity slopes during L2/3 deactivation were evaluated using two methods of sorting: 

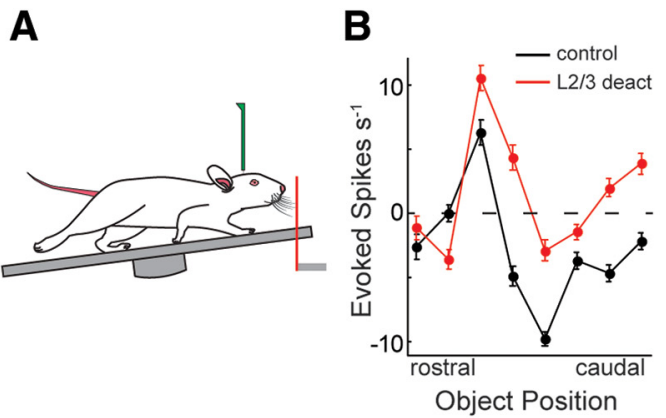

C
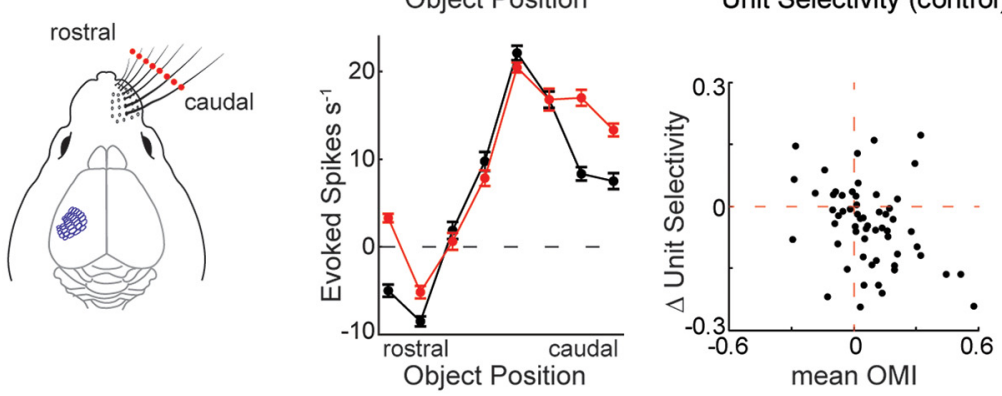

D
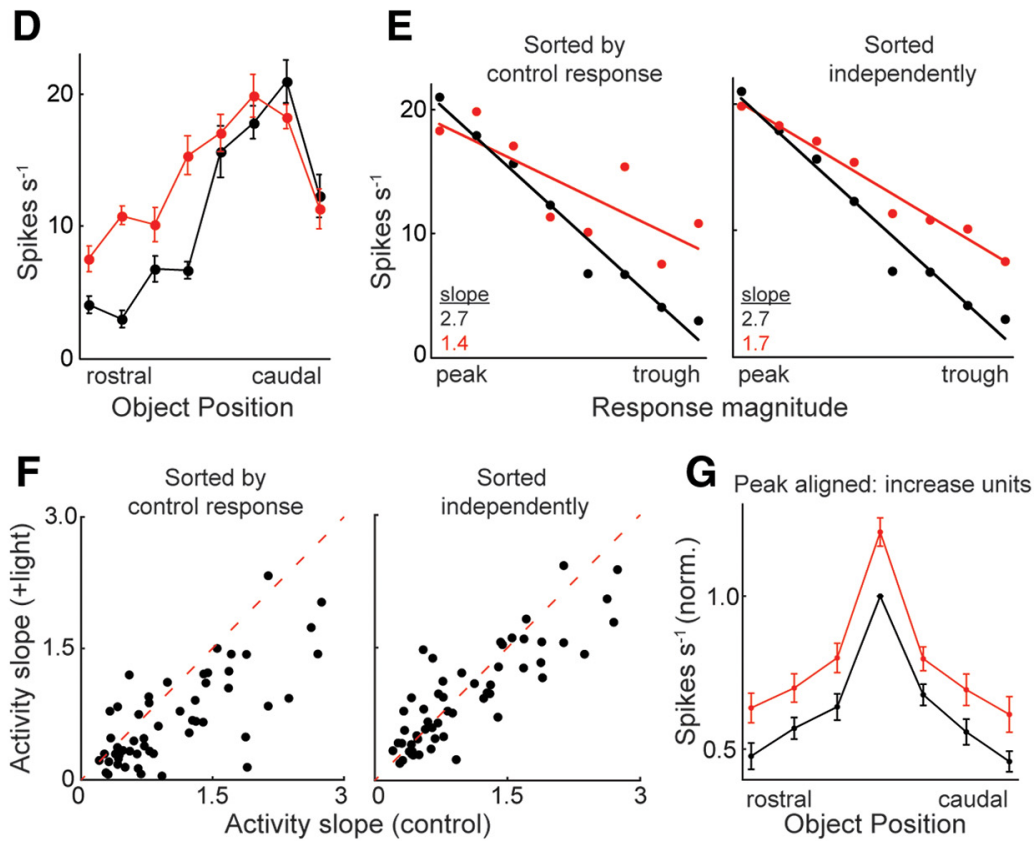

Figure 7. $L 2 / 3$ increases the selectivity and range of $L 5 R S$ receptive fields. $\boldsymbol{A}$, Experimental set-up: head-fixed, running mice with their whisker pad intact palpated an object that was placed at eight different locations within their whisking field. $\boldsymbol{B}$, Spatial tuning curves and rasters of two L5 RS neurons. Note the loss of touch suppression and the greater increase in spike rate in the surround regions of their receptive fields. C, Scatter plot comparing the stimulus selectivity of L5 RS units during control stimulation versus $\mathrm{L} 2 / 3$ deactivation (top, + light). $\mathrm{L} 2 / 3$ deactivation significantly reduces stimulus selectivity $(p=0.001, n=58$, Wilcoxon signed-rank test). Scatter plot of the L5 RS population comparing the mean 0MI to the change in unit selectivity (bottom). $\boldsymbol{D}$, Example L5 RS neuron with a tuning peak at the caudal region of the whisker field and a trough at the rostral region. $\boldsymbol{E}$, The activity slopes of the example unit in $\boldsymbol{D}$. Slopes were calculated after sorting in descending order relative to the magnitude of the control response (left) or sorting independently for both control and L2/3 deactivation conditions (right). $F$, Scatter plots of the L5 RS population comparing the activity slopes during control stimulation versus $L 2 / 3$ deactivation. $L 2 / 3$ deactivation significantly reduced the steepness of the slope ( $p<0.0001$ : left; $p=0.02$ : right, Wilcoxon signed-rank test, $n=58$ ). G, Mean \pm SEM tuning curves of the population of L5RS neurons that displayed an increase in activity during $\mathrm{L} 2 / 3$ deactivation $(n=41)$. Firing rates in each condition were aligned to their own peak and normalized to the largest control response. Error bars are the standard error of the mean.

(1) same order as the control firing rates and (2) independent descending order (Fig. 7E). Sorting both conditions by the control firing rates evaluates changes to receptive field shape, whereas independent sorting evaluates the total range of activity. Across the population, the activity slope was dramatically re- duced during L2/3 deactivation when sorted according to the control response (Fig. $7 F ; p<0.001, n=58$, Wilcoxon signed-rank test) and was more moderately reduced when sorted independently (Fig. $7 F ; p=0.02, n=58$, Wilcoxon signed-rank test). In addition, the depth of modulation between the peak and trough of the receptive field significantly decreased during L2/3 deactivation $(p<$ $0.001, n=58$, Wilcoxon signed-rank test). Therefore, L2/3 deactivation dramatically disrupts the shape of receptive fields in L5 and contracts their relative range of activity. However, the absolute range of firing rate $(\max -\min )$ did not significantly change as a population $(p=$ $0.27, n=58$, Wilcoxon signed-rank test), indicating that selectivity changes in many units were additive across space, as visualized by the population averaged and peakaligned tuning curves (Fig. 7G). The decrease in spatial selectivity seems unlikely to be explained by a complementary change in the spatial tuning of L5 FS neurons, as is true when optogenetically suppressing L4 (Pluta et al., 2015), because there was no consistent effect on their stimulus selectivity (Fig. 8; $p=1.0, n=$ 40 , Wilcoxon signed-rank test).

\section{Discussion}

The data presented here demonstrate that L2/3 has a significant impact on neural activity in L5. Contrary to the conventional notion, the net impact of L2/3 activity on the plurality of L5 neurons was suppression. During optogenetic stimulation of L2/3, nearly twice as many L5 units were suppressed as were facilitated. Conversely, during natural sensory stimulation, optogenetic suppression of L2/3 facilitated twice as many L5 neurons as it suppressed. Optogenetic activation experiments must be interpreted with caution, as ChR2-mediated photostimulation inherently generates nonphysiological patterns of activity in the stimulated population. However, our data with optogenetic suppression of L2/3 sensory responses, which show nearly the opposite effects of activation, support this conclusion. Moreover, in our ChR2 experiments, we attempted to generate firing rates in L2/3 neurons that were comparable with those observed during active touch, and we stress that our ramplight stimuli avoided the extreme levels of hyper-synchronization of ChR2-expressing neurons one may obtain during brief, high intensity pulses of light.

Importantly, the translaminar suppression from L2/3 to L5 sharpened the representation of space in L5 pyramidal neurons by preferentially acting at nonpreferred spatial locations. $\mathrm{SST}^{+}$ 
interneurons likely play an important role in mediating these effects. It should be noted that the extracellular recording methods used in this study may bias the detection of units with firing rates higher than the mean of the true underlying distribution. However, because higher firing rate neurons are the most effective at recruiting polysynaptic inhibition through $\mathrm{SST}^{+}$neurons (Kapfer et al., 2007; Silberberg and Markram, 2007; Berger et al., 2010), the units we did observe are likely to be among the most impactful in the circuit.

Our data also support the notion that much of the activating drive to L5 during touch must come from sources beyond L2/3 PCs. Many of the L5 neurons that were suppressed by L2/3 were facilitated by touch in a restricted portion of tactile space. These neurons may broadly integrate translaminar suppression in combination with a different source of facilitating input, possibly directly from the thalamus (Constantinople and Bruno, 2013). The relative strength and breadth of these opposing inputs (e.g., thalamus vs L2/3) may determine the size and shape of spatial receptive fields, which are considerably diverse in the cortex (Gilbert, 1977; Manns et al., 2004; Runyan et al., 2010; Pluta et al., 2017). By using intracortical inhibition to balance ascending thalamic and descending intracortical excitation, L5 receptive fields can be dynamically shaped, possibly based on behavioral demands (Polack et al., 2013).

Multiple circuit models could explain our data. First, much of the effects could depend on horizontally arborizing axons of L2/3 within L5 (Feldmeyer, 2012), which may recruit lateral inhibition via $\mathrm{SST}^{+}$neurons. SST ${ }^{+}$neurons can also integrate recurrent input directly from L5 neurons (Silberberg and Markram, 2007). Horizontal circuits within L2/3, such as local recruitment of $\mathrm{SST}^{+}$neurons that synapse onto L5 dendrites, could also contribute to the suppression of L5. Finally, circuits residing within the same column as the recorded L5 neurons could be vital. Distinguishing among these possibilities will require future work that uses techniques, such as spatially precise two-photon optogenetics, to activate or inactivate identified cortical neurons in discrete sets of whisker columns (Mardinly et al., 2018).

Both our in vivo and in vitro data in the barrel cortex indicate that $\mathrm{L} 2 / 3$ recruits inhibition via SST ${ }^{+}$interneurons to suppress L5, conceptually consistent with in vitro brain slice data from the motor and visual cortices (Apicella et al., 2012; Jiang et al., 2015). Although we do not provide direct evidence that L2/3 pyramidal neurons monosynaptically recruit $\mathrm{L}_{5 \mathrm{SST}}{ }^{+}$neurons to suppress L5 pyramidal neurons in the barrel cortex, the synaptic connections making this possible have been observed in brain slices of the frontal cortex (Otsuka and Kawaguchi, 2009). Although these data implicate $\mathrm{SST}^{+}$in translaminar integration, their functional role of enhancing translaminar suppression and moderating translaminar facilitation was previously unknown. Future research is needed to delineate the laminar distribution of L2/3mediated SST ${ }^{+}$inhibition onto L5. Our data argue against FS/
B
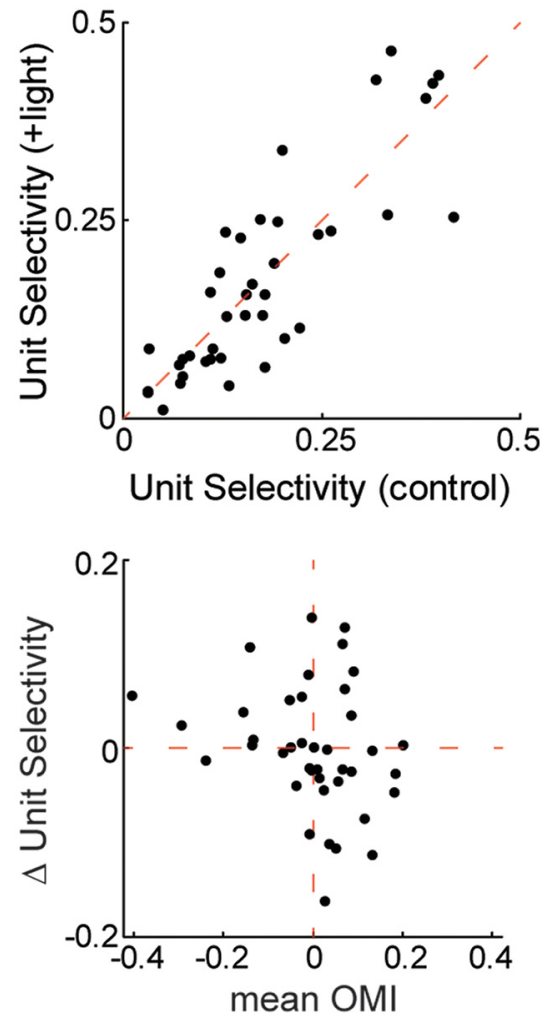

Figure 8. $\quad \mathrm{L} 2 / 3$ does not consistently alter the spatial selectivity of $L 5 F S$ units. $A$, Spatial tuning curves from two $L 5 F S$ neurons during control stimulation and $\mathrm{L} 2 / 3$ deactivation. $\boldsymbol{B}$, Scatter plot comparing the selectivity of $\mathrm{L} 5 \mathrm{FS}$ neurons during control stimulation and $\mathrm{L} 2 / 3$ deactivation ( $p=1.0$, Wilcoxon signed-rank test, $n=40$, top). Scatter plot relating the change in mean $0 \mathrm{MI}$ to the change in unit selectivity during $\mathrm{L} 2 / 3$ deactivation (bottom). Error bars are the standard error of the mean.

$\mathrm{PV}^{+}$interneurons as the primary mediator of L2/3's suppression of L5. In our previous publication, using identical techniques, we showed that L4 suppresses L5 by facilitating the vast majority of FS/PV ${ }^{+}$interneurons (Pluta et al., 2015). In contrast, the current study reveals that L2/3 suppresses as many FS units as it facilitates, with no net change in activity across the population. Notably, not all FS units are $\mathrm{PV}^{+}$, with a small minority corresponding to excitatory neurons and others possibly thin-spiking $\mathrm{SST}^{+}$ neurons (Moore and Wehr, 2013; Muñoz et al., 2014). Future research identifying functionally connected translaminar neuronal ensembles could help clarify the heterogeneous effects we observed.

In some L5 units ( $\sim 40 \%)$, we observed no significant change in firing rate while manipulating $\mathrm{L} 2 / 3$. This result is expected given that different subtypes of L5 neurons integrate divergent streams of input, sometimes preferentially from either subcortical or long-range cortical areas (Wright and Fox, 2010; Mao et al., 2011; Joshi et al., 2015; Kim et al., 2015; Kinnischtzke et al., 2016). Technological innovations that combine trans-synaptic labeling of projection neurons, multiphoton imaging of deep tissue, and layer-specific optogenetic manipulations could determine whether long-range connectivity patterns predict functional relationships within the intracortical microcircuit.

Layer 5 PCs fire action potentials at high rates in the absence of external stimuli, distinct from the sparse activity patterns found in layers 2-4 (Simons and Carvell, 1989; Brecht and Sakmann, 2002; Kerr et al., 2007; Jadhav et al., 2009; Crochet et al., 2011; Barth and Poulet, 2012; Hires et al., 2015). While the precise advantage of maintaining such high ongoing firing rates remains 
a mystery, this feature is essential for enabling sensory suppression to enhance stimulus selectivity and expand dynamic range, both within single units and across a sensory map (Tantirigama et al., 2017). Our results are the first to demonstrate that L2/3 activity is important for this unique form of sensory coding in L5.

Experimental manipulations testing the causal relationship between cortical layers have traditionally been very challenging and have only recently become tenable with modern optogenetic techniques (Olsen et al., 2012; Pluta et al., 2015; Guo et al., 2017). The previous study to causally test the impact of $\mathrm{L} 2 / 3$ on sensory responses L5, albeit using cryogenic deactivation, found little to no effect on the firing rate or temporal pattern of activity (Schwark et al., 1986), although this was conducted in the anesthetized cat. In the present study, we focused on activity during alert, actively sensing conditions, where many neurons in L2/3 of the somatosensory cortex are highly responsive to touch (Pluta et al., 2017). The disinhibitory effects we observed during L2/3 deactivation could be obscured by anesthesia, which greatly alters interneuron and spontaneous activity (Armstrong-James and George, 1988; de Kock and Sakmann, 2008; Adesnik et al., 2012; Sellers et al., 2013; McGinley et al., 2015; Muñoz et al., 2017).

Overall, our data support what emerges as a surprising principle of cortical dynamics: the net impact of both superficial layers on L5 is primarily suppression, rather than facilitation. This translaminar suppression sharpens stimulus representations in L5, but through different translaminar inhibitory mechanisms: $\mathrm{L} 4$ relies primarily on $\mathrm{L} 5 \mathrm{FS} / \mathrm{PV}^{+}$neurons, whereas $\mathrm{L} 2 / 3$, at least in part, depends on $\mathrm{SST}^{+}$neurons. Together, these data highlight the importance of translaminar inhibitory circuits in dynamically controlling the sign and spread of cortical activity in the primary output layer.

\section{References}

Adesnik H, Scanziani M (2010) Lateral competition for cortical space by layer-specific horizontal circuits. Nature 464:1155-1160.

Adesnik H, Bruns W, Taniguchi H, Huang ZJ, Scanziani M (2012) A neural circuit for spatial summation in visual cortex. Nature 490:226-231.

Apicella AJ, Wickersham IR, Seung HS, Shepherd GM (2012) Laminarly orthogonal excitation of fast-spiking and low-threshold-spiking interneurons in mouse motor cortex. J Neurosci 32:7021-7033.

Armstrong-James M, George MJ (1988) Influence of anesthesia on spontaneous activity and receptive field size of single units in rat Sm1 neocortex. Exp Neurol 99:369-387.

Armstrong-James M, Fox K, Das-Gupta A (1992) Flow of excitation within rat barrel cortex on striking a single vibrissa. J Neurophysiol 68:13451358.

Bala AD, Spitzer MW, Takahashi TT (2003) Prediction of auditory spatial acuity from neural images on the owl's auditory space map. Nature 424: 771-774.

Barth AL, Poulet JF (2012) Experimental evidence for sparse firing in the neocortex. Trends Neurosci 35:345-355.

Berger TK, Silberberg G, Perin R, Markram H (2010) Brief bursts selfinhibit and correlate the pyramidal network. PLoS Biol 8:e1000473.

Brecht M, Sakmann B (2002) Dynamic representation of whisker deflection by synaptic potentials in spiny stellate and pyramidal cells in the barrels and septa of layer 4 rat somatosensory cortex. J Physiol 543:49-70.

Butts DA, Goldman MS (2006) Tuning curves, neuronal variability, and sensory coding. PLoS Biol 4:639-646.

Celio MR (1986) Parvalbumin in most gamma-aminobutyric acidcontaining neurons of the rat cerebral cortex. Science 231:995-997.

Clack NG, O'Connor DH, Huber D, Petreanu L, Hires A, Peron S, Svoboda K, Myers EW (2012) Automated tracking of whiskers in videos of head fixed rodents. PLoS Comput Biol 8:e1002591.

Constantinople CM, Bruno RM (2011) Effects and mechanisms of wakefulness on local cortical networks. Neuron 69:1061-1068.

Constantinople CM, Bruno RM (2013) Deep cortical layers are activated directly by thalamus. Science 340:1591-1594.
Crochet S, Poulet JF, Kremer Y, Petersen CC (2011) Synaptic mechanisms underlying sparse coding of active touch. Neuron 69:1160-1175.

Curtis JC, Kleinfeld D (2009) Phase-to-rate transformations encode touch in cortical neurons of a scanning sensorimotor system. Nat Neurosci 12:492-501.

de Kock CP, Sakmann B (2008) High frequency action potential bursts $(>$ or $=100 \mathrm{~Hz})$ in L2/3 and L5B thick tufted neurons in anaesthetized and awake rat primary somatosensory cortex. J Physiol 586:3353-3364.

de Kock CP, Sakmann B (2009) Spiking in primary somatosensory cortex during natural whisking in awake head-restrained rats is cell-type specific. Proc Natl Acad Sci U S A 106:16446-16450.

Ego-Stengel V, Mello e Souza T, Jacob V, Shulz DE (2005) Spatiotemporal characteristics of neuronal sensory integration in the barrel cortex of the rat. J Neurophysiol 93:1450-1467.

Feldmeyer D (2012) Excitatory neuronal connectivity in the barrel cortex. Front. Neuroanat 6:24.

Ghazanfar AA, Nicolelis MA (1999) Spatiotemporal properties of layer V neurons of the rat primary somatosensory cortex. Cereb Cortex 9:348-361

Gilbert CD (1977) Laminar differences in receptive field properties of cells in cat primary visual cortex. J Physiol 268:391-421.

Guo W, Clause AR, Barth-Maron A, Polley DB (2017) A corticothalamic circuit for dynamic switching between feature detection and discrimination. Neuron 95:180-194.e5.

Hakim R, Shamardani K, Adesnik H (2018) A neural circuit for gammaband coherence across the retinotopic map in mouse visual cortex. Elife 7:e29569.

Hill DN, Mehta SB, Kleinfeld D (2011) Quality metrics to accompany spike sorting of extracellular signals. J Neurosci 31:8699-8705.

Hires SA, Gutnisky DA, Yu J, O'Connor DH, Svoboda K (2015) Low-noise encoding of active touch by layer 4 in the somatosensory cortex. Elife 4.

Hooks BM, Hires SA, Zhang YX, Huber D, Petreanu L, Svoboda K, Shepherd GM (2011) Laminar analysis of excitatory local circuits in vibrissal motor and sensory cortical areas. PLoS Biol. 9:e1000572.

Ito M (1992) Simultaneous visualization of cortical barrels and horseradish peroxidase-injected layer 5b vibrissa neurones in the rat. J Physiol 454: 247-265.

Jadhav SP, Wolfe J, Feldman DE (2009) Sparse temporal coding of elementary tactile features during active whisker sensation. Nat Neurosci 12:792800

Jiang X, Shen S, Cadwell CR, Berens P, Sinz F, Ecker AS, Patel S, Tolias AS (2015) Principles of connectivity among morphologically defined cell types in adult neocortex. Science 350:aac9462.

Joshi A, Middleton JW, Anderson CT, Borges K, Suter BA, Shepherd GM, Tzounopoulos T (2015) Cell-specific activity-dependent fractionation of layer $2 / 3 \rightarrow 5 \mathrm{~B}$ excitatory signaling in mouse auditory cortex. J Neurosci 35:3112-3123.

Kapfer C, Glickfeld LL, Atallah BV, Scanziani M (2007) Supralinear increase of recurrent inhibition during sparse activity in the somatosensory cortex. Nat Neurosci 10:743-753.

Kawaguchi Y, Katsumaru H, Kosaka T, Heizmann CW, Hama K (1987) Fast spiking cells in rat hippocampus (CA1 region) contain the calciumbinding protein parvalbumin. Brain Res 416:369-374.

Kerr JN, de Kock CP, Greenberg DS, Bruno RM, Sakmann B, Helmchen F (2007) Spatial organization of neuronal population responses in layer 2/3 of rat barrel cortex. J Neurosci 27:13316-13328.

Kim EJ, Juavinett AL, Kyubwa EM, Jacobs MW, Callaway EM (2015) Three types of cortical layer 5 neurons that differ in brain-wide connectivity and function. Neuron 88:1253-1267.

Kinnischtzke AK, Fanselow EE, Simons DJ (2016) Target-specific M1 inputs to infragranular S1 pyramidal neurons. J Neurophysiol 116:12611274 .

Krupa DJ, Wiest MC, Shuler MG, Laubach M, Nicolelis MA (2004) Layerspecific somatosensory cortical activation during active tactile discrimination. Science 304:1989-1992.

Lefort S, Tomm C, Floyd Sarria JC, Petersen CC (2009) The excitatory neuronal network of the $\mathrm{C} 2$ barrel column in mouse primary somatosensory cortex. Neuron 61:301-316.

Manns ID, Sakmann B, Brecht M (2004) Sub- and suprathreshold receptive field properties of pyramidal neurones in layers $5 \mathrm{~A}$ and $5 \mathrm{~B}$ of rat somatosensory barrel cortex. J Physiol 556:601-622.

Mao T, Kusefoglu D, Hooks BM, Huber D, Petreanu L, Svoboda K (2011) 
Long-range neuronal circuits underlying the interaction between sensory and motor cortex. Neuron 72:111-123.

Mardinly AR, Oldenburg IA, Pégard NC, Sridharan S, Lyall EH, Chesnov K, Brohawn SG, Waller L, Adesnik H (2018) Precise multimodal optical control of neural ensemble activity. Nat Neurosci 21:881-893.

Markram H, Toledo-Rodriguez M, Wang Y, Gupta A, Silberberg G, Wu C (2004) Interneurons of the neocortical inhibitory system. Nat Rev Neurosci 5:793-807.

McGinley MJ, Vinck M, Reimer J, Batista-Brito R, Zagha E, Cadwell CR, Tolias AS, Cardin JA, McCormick DA (2015) Waking state: rapid variations modulate neural and behavioral responses. Neuron 87:1143-1161.

Moore AK, Wehr M (2013) Parvalbumin-expressing inhibitory interneurons in auditory cortex are well-tuned for frequency. J Neurosci 33:13713-13723.

Muñoz W, Tremblay R, Rudy B (2014) Channelrhodopsin-assisted patching: in vivo recording of genetically and morphologically identified neurons throughout the brain. Cell Rep 9:2304-2316.

Muñoz W, Tremblay R, Levenstein D, Rudy B (2017) Layer-specific modulation of neocortical dendritic inhibition during active wakefulness. Science 355:954-959.

Naka A, Adesnik H (2016) Inhibitory circuits in cortical layer 5. Front Neural Circuits 10:35.

O'Connor DH, Peron SP, Huber D, Svoboda K (2010) Neural activity in barrel cortex underlying vibrissa-based object localization in mice. Neuron 67:1048-1061.

Oliva AA Jr, Jiang M, Lam T, Smith KL, Swann JW (2000) Novel hippocampal interneuronal subtypes identified using transgenic mice that express green fluorescent protein in GABAergic interneurons. J Neurosci 20:3354-3368.

Olsen SR, Bortone DS, Adesnik H, Scanziani M (2012) Gain control by layer six in cortical circuits of vision. Nature 483:47-52.

Otsuka T, Kawaguchi Y (2009) Cortical inhibitory cell types differentially form intralaminar and interlaminar subnetworks with excitatory neurons. J Neurosci 29:10533-10540.

Petreanu L, Mao T, Sternson SM, Svoboda K (2009) The subcellular organization of neocortical excitatory connections. Nature 457:1142-1145.

Pluta S, Naka A, Veit J, Telian G, Yao L, Hakim R, Taylor D, Adesnik H (2015) A direct translaminar inhibitory circuit tunes cortical output. Nat Neurosci 18:1631-1640.

Pluta SR, Lyall EH, Telian GI, Ryapolova-Webb E, Adesnik H (2017) Surround integration organizes a spatial map during active sensation. Neuron 94:1220-1233.e5.

Polack PO, Friedman J, Golshani P (2013) Cellular mechanisms of brain state-dependent gain modulation in visual cortex. Nat Neurosci 16: 1331-1339.

Qi HX, Reed JL, Franca JG, Jain N, Kajikawa Y, Kaas JH (2016) Chronic recordings reveal tactile stimuli can suppress spontaneous activity of neurons in somatosensory cortex of awake and anesthetized primates. J Neurophysiol 115:2105-2123.

Ramirez A, Pnevmatikakis EA, Merel J, Paninski L, Miller KD, Bruno RM
(2014) Spatiotemporal receptive fields of barrel cortex revealed by reverse correlation of synaptic input. Nat Neurosci 17:866-875.

Reyes A, Lujan R, Rozov A, Burnashev N, Somogyi P, Sakmann B (1998) Target-cell-specific facilitation and depression in neocortical circuits. Nat Neurosci 1:279-285.

Reyes-Puerta V, Sun JJ, Kim S, Kilb W, Luhmann HJ (2015) Laminar and columnar structure of sensory-evoked multineuronal spike sequences in adult rat barrel cortex in vivo. Cereb Cortex 25:2001-2021.

Rolls ET (2016) Cortical coding. Lang Cogn Neurosci 44:1-14.

Rolls ET, Tovee MJ (1995) Sparseness of the neuronal representation of stimuli in the primate temporal visual cortex. J Neurophysiol 73:713-726.

Runyan CA, Schummers J, Van Wart A, Kuhlman SJ, Wilson NR, Huang ZJ, Sur M (2010) Response features of parvalbumin-expressing interneurons suggest precise roles for subtypes of inhibition in visual cortex. Neuron 67:847-857.

Schwark HD, Malpeli JG, Weyand TG, Lee C (1986) Cat area 17: II. Response properties of infragranular layer neurons in the absence of supragranular layer activity. J Neurophysiol 56:1074-1087.

Sellers KK, Bennett DV, Hutt A, Fröhlich F (2013) Anesthesia differentially modulates spontaneous network dynamics by cortical area and layer. J Neurophysiol 110:2739-2751.

Seriès P, Latham PE, Pouget A (2004) Tuning curve sharpening for orientation selectivity: coding efficiency and the impact of correlations. Nat Neurosci 7:1129-1135.

Shimegi S, Ichikawa T, Akasaki T, Sato H (1999) Temporal characteristics of response integration evoked by multiple whisker stimulations in the barrel cortex of rats. J Neurosci 19:10164-10175.

Silberberg G, Markram H (2007) Disynaptic inhibition between neocortical pyramidal cells mediated by Martinotti cells. Neuron 53:735-746.

Simons DJ, Carvell GE (1989) Thalamocortical response transformation in the rat vibrissa/barrel system. J Neurophysiol 61:311-330.

Sofroniew NJ, Cohen JD, Lee AK, Svoboda K (2014) Natural whiskerguided behavior by head-fixed mice in tactile virtual reality. J Neurosci 34:9537-9550.

Sofroniew NJ, Vlasov YA, Hires SA, Freeman J, Svoboda K (2015) Neural coding in barrel cortex during whisker-guided locomotion. Elife 4:e12559.

Takahashi N, Oertner TG, Hegemann P, Larkum ME (2016) Active cortical dendrites modulate perception. Science 354:1587-1590.

Tantirigama ML, Huang HH, Bekkers JM (2017) Spontaneous activity in the piriform cortex extends the dynamic range of cortical odor coding. Proc Natl Acad Sci U S A 114:2407-2412.

Vinje WE, Gallant JL (2000) Sparse coding and decorelation in primary visual cortex during natural stimulation. Science 287:1273-1276.

Wright N, Fox K (2010) Origins of cortical layer V surround receptive fields in the rat barrel cortex. J Neurophysiol 103:709-724.

Xiang Z, Huguenard JR, Prince DA (2002) Synaptic inhibition of pyramidal cells evoked by different interneuronal subtypes in layer $\mathrm{V}$ of rat visual cortex. J Neurophysiol 88:740-750. 